\title{
Modular Categories*
}

\author{
M. Müger \\ IMAPP, Radboud University Nijmegen \\ The Netherlands
}

February 13, 2012

\section{Introduction}

Modular categories, as well as the (possibly) more general non-degenerate braided fusion categories, are braided tensor categories that are linear over a field and satisfy some natural additional axioms, like existence of duals, semisimplicity, finiteness, and an important non-degeneracy condition. (Precise definitions will be given later.) There are several reasons to study modular categories:

- As will hopefully become clear, they are rather interesting mathematical structures in themselves, well worth being studied for intrinsic reasons. For example, there are interesting number theoretic aspects.

- Among the braided fusion categories, modular categories are the opposite extreme of the symmetric fusion categories, which are well known to be closely related to finite groups. Studying these two extreme cases is also helpful for understanding and classifying those braided fusion categories that are neither symmetric nor modular.

- Modular categories serve as input datum for the Reshetikhin-Turaev construction of topological quantum field theories in $2+1$ dimensions and therefore give rise to invariants of smooth 3-manifolds. This goes some way towards making Witten's interpretation of the Jones polynomial via Chern-Simons QFT rigorous. (But since there still is no complete rigorous non-perturbative construction of the Chern-Simons QFTs by conventional quantum field theory methods, there also is no proof of their equivalence to the RT-TQFTs constructed using the representation theory of quantum groups.)

- Modular categories arise as representation categories of loop groups and, more generally, of rational chiral conformal quantum field theories. In chiral CQFT, the field theory itself, its representation category, and the conformal characters form a remarkably tightly connected structure.

- Also certain massive quantum field theories and quantum spin systems in two spatial dimensions lead to modular categories, e.g. Kitaev's 'toric code'.

- The recent topological approaches to quantum computing, while differing in details, all revolve around the notion of modular categories.

As the above list indicates, modular categories - and related mathematical subjects like representation theory of loop groups and of quantum groups at root-of-unity deformation parameter - represent one of the most fruitful places of interaction of 'pure' mathematics and mathematical physics. (While modular categories play a certain rôle in string theory via their importance for rational conformal field theories, the author believes that their appearance in massive field theories and spin models may ultimately turn out to be of larger relevance for realistic physics.)

This article assumes a certain familiarity with category theory, including monoidal (=tensor) categories. Concerning braided categories, only the definition, for which we refer to Majid's contribution, will be assumed. Our standard reference for category theory is [59]. For a broader survey of some related matters concerning tensor categories, cf. also [72].

${ }^{*}$ To appear in: C. Heunen, M. Sadrzadeh, E. Grefenstette (eds.): Compositional methods in quantum physics and linguistics, Oxford University Press, 2012. 


\section{Categories}

We limit ourselves to recalling some basic definitions. A category is called an Ab-category if it is enriched over (the symmetric tensor category Ab of) abelian groups. I.e., all hom-sets come with abelian group structures and the composition o of morphisms is a homomorphism w.r.t. both arguments. An Ab-category is called additive if it has a zero object and every pair of objects has a direct sum. If $k$ is a field (or more generally, a commutative unital ring) then a category $\mathcal{C}$ is called $k$-linear if all hom-sets are $k$-vector spaces (or $k$-modules) and $\circ$ is bilinear. An object $X$ is called simple if every monic morphism $Y \hookrightarrow X$ is an isomorphism and absolutely simple if End $X \cong k \mathrm{id}_{X}$. If $k$ is an algebraically closed field, as we will mostly assume, the two notions coincide. A $k$-linear category is semisimple if every object is a finite direct sum of simple objects. A semisimple category is called finite if the number of isomorphism classes of simple objects is finite. (There is a notion of finiteness for non-semisimple categories, cf. [34, but we will not need it.) A positive $*$-operation on a $\mathbb{C}$-linear category $\mathcal{C}$ is a contravariant endofunctor of $\mathcal{C}$ that acts like the identity on objects, is involutive $(* *=\mathrm{id})$ and anti-linear. (Dropping the $\mathbb{C}$-linearity, one arrives at the notion of a dagger-category.) $\mathrm{A} *$-operation is called positive if $s^{*} \circ s=0$ implies $s=0$. A category equipped with a (positive) $*$-operation is called hermitian (unitary). A unitary category with finite-dimensional hom-spaces and splitting idempotents is semisimple (since finite dimensional algebras with positive $*$-operation are semisimple, thus multi-matrix algebras).

\section{Tensor categories}

We assume familiarity with the basics of tensor (=monoidal) categories, including symmetric ones. We therefore limit the discussion to (i) issues of duality in not necessarily braided tensor categories, (ii) fusion categories and (iii) module categories vs. categories of modules over an algebra in a tensor category.

\subsection{Duality}

In the following, we will state definitions and results for strict tensor categories, but everything can easily be adapted to the non-strict case.

3.1 Definition Let $\mathcal{C}$ be a tensor category and let $X, Y \in \mathcal{C}$. If there exist morphisms $e: Y \otimes X \rightarrow \mathbf{1}, d: \mathbf{1} \rightarrow$ $X \otimes Y$ satisfying

$$
\left(\operatorname{id}_{X} \otimes e\right) \circ\left(d \otimes \operatorname{id}_{X}\right)=\operatorname{id}_{X}, \quad\left(e \otimes \operatorname{id}_{Y}\right) \circ\left(\operatorname{id}_{Y} \otimes d\right)=\operatorname{id}_{Y},
$$

then $Y$ is called a left dual of $X$ and $X$ is a right dual of $Y$. If every object has a left (right) dual, we say that $\mathcal{C}$ has left (right) duals.

It is easy to prove that duals are unique up to isomorphism: If $Y, Y^{\prime}$ are both left (or both right) duals of $X$ then $Y \cong Y^{\prime}$. This justifies writing ${ }^{\vee} X\left(X^{\vee}\right)$ for a left (right) dual of $X$. However, if $X$ admits a left dual ${ }^{\vee} X$ and a right dual $X^{\vee}$, it may or may not be the case that ${ }^{\vee} X \cong X^{\vee}$. Clearly, a left dual ${ }^{\vee} X$ of $X$ is also a right dual of $X$ if and only if $X$ is a left dual of ${ }^{\vee} X$, thus if and only if $X$ is isomorphic to its second left dual ${ }^{\vee}{ }^{\vee} X$. If ${ }^{\vee} X \cong X^{\vee}$ holds, we say that $X$ has a two-sided dual and mostly write $\bar{X}$ rather than ${ }^{\vee} X$ or $X^{\vee}$. If all objects have two-sided duals, we say that $\mathcal{C}$ has two-sided duals.

There are three situations where duals, to the extent that they exist, are automatically two-sided:

(i) $\mathcal{C}$ is hermitian: If $\left({ }^{\vee} X, e_{X}, d_{X}\right)$ defines a left dual of $X$, one finds that $\left({ }^{\vee} X, d_{X}^{*}, e_{X}^{*}\right)$ is a right dual. In this situation, some authors talk about conjugates rather than duals and give slightly different axioms, cf. [57.

(ii) $\mathcal{C}$ is braided, cf. Section 4.5 .

(iii) $\mathcal{C}$ is semisimple with simple unit, cf. [32, Proposition 2.1].

If $\mathcal{C}$ is linear over a field $k$ and the unit $\mathbf{1}$ is absolutely simple, we can and will use the bijection $k \rightarrow$ End $\mathbf{1}, c \mapsto c \mathrm{id}_{\mathbf{1}}$ to identify End $\mathbf{1}$ with $k$. If $X \in \mathcal{C}$ is absolutely simple and has a two-sided dual $\bar{X}$, one defines 'squared dimension' $d^{2}(X)=\left(e^{\prime} \circ d\right)\left(e \circ d^{\prime}\right)$, which is easily seen to be independent of the choices of the duality morphisms $e, d, e^{\prime}, d^{\prime}$, cf. [65]. If $X, Y$ and $X \otimes Y$ are absolutely simple, one has $d^{2}(X \otimes Y)=d^{2}(X) d^{2}(Y)$. If $\mathcal{C}$ is semisimple and finite, we define $\operatorname{dim} \mathcal{C}=\sum_{i} d^{2}\left(X_{i}\right)$, where $\left\{X_{i}\right\}$ is a complete family of simple objects.

In the $k$-linear case, one would like to have a dimension function $X \mapsto d(X)$ that is additive and multiplicative. If $\mathcal{C}$ is semisimple and finite, this can be done using Perron-Frobenius theory, cf. the next section. Another approach strengthens the requirement of existence of duals by introducing a new piece of structure: 
3.2 Definition $A$ left duality on a tensor category is an assignment $X \mapsto\left({ }^{\vee} X, e_{X}, d_{X}\right)$, where ${ }^{\vee} X$ is a left dual of $X$, the morphisms $e_{X}:{ }^{\vee} X \otimes X \rightarrow \mathbf{1}, d_{X}: \mathbf{1} \rightarrow X \otimes{ }^{\vee} X$ satisfying the above identities. Similarly, a right duality is an assignment $X \mapsto\left(X^{\vee}, e_{X}^{\prime}, d_{X}^{\prime}\right)$ where the morphisms $e_{X}^{\prime}: X \otimes X^{\vee} \rightarrow \mathbf{1}, d_{X}^{\prime}: \mathbf{1} \rightarrow X^{\vee} \otimes X$ establish $X^{\vee}$ as a right dual of $X$.

If $\mathcal{C}$ is a tensor category with a chosen left duality $X \mapsto\left({ }^{\vee} X, e_{X}, d_{X}\right)$ and $f: X \rightarrow Y$ then

$$
{ }^{\vee} f=\left(e_{Y} \otimes \operatorname{id}_{\vee_{X}}\right) \circ\left(\operatorname{id}_{v_{Y}} \otimes f \otimes \operatorname{id}_{\vee_{X}}\right) \circ\left(\operatorname{id}_{X} \otimes d_{X}\right):{ }^{\vee} Y \rightarrow{ }^{\vee} X
$$

extends $X \mapsto{ }^{\vee} X$ to a contravariant endofunctor ${ }^{\vee}-$ of $\mathcal{C}$. Since ${ }^{\vee} Y \otimes{ }^{\vee} X$ is a left dual of $X \otimes Y,{ }^{\vee}-$ can be considered as a (covariant) tensor functor $\mathcal{C} \rightarrow \mathcal{C}^{\text {op,rev }}$, where $\mathcal{C}^{\text {rev }}$ coincides with $\mathcal{C}$ as a category, but has the reversed tensor product $X \otimes^{\text {rev }} Y=Y \otimes X$. (Similarly for $-{ }^{\vee}$.) If ${ }^{\vee}$ - is a left duality and $\mathcal{C}$ has two-sided duals, one can find a natural isomorphism $\gamma:$ id $\rightarrow^{\vee \vee}$ - Since this is of little use unless $\gamma$ is monoidal, one defines:

3.3 Definition A pivotal category [37, 38] is a tensor category together with a left duality ${ }^{\vee}$ - and a monoidal natural isomorphism $\gamma:$ id $\rightarrow{ }^{\vee v_{-}}$.

3.4 Remark 1. Categories equipped with left and/or right dualities are often called rigid or autonomous, and pivotal categories are also called sovereign. We will avoid all these terms. The categories we consider will either have the property of possessing two-sided duals (without given duality structures) or be equipped with pivotal or spherical structures.

2. For a general tensor category with two-sided duals, there is little a priori reason to expect the existence of a pivotal structure, but see Theorems 3.6 and 3.7 below.

3.5 Definition Let $\mathcal{C}$ be a strict pivotal category with left duality $X \mapsto\left(\bar{X}, e_{X}, d_{X}\right)$ and monoidal natural isomorphism $\left(\alpha_{X}: X \rightarrow \overline{\bar{X}}\right)_{X \in \mathcal{C}}$. For $X \in \mathcal{C}$ and $s \in$ End $X$, define the left and right traces of $s$ by

$$
\begin{aligned}
& \operatorname{tr}_{X}^{L}(s)=e_{X} \circ\left(\operatorname{id}_{\bar{X}} \otimes\left(s \circ \alpha_{X}^{-1}\right)\right) \circ d_{\bar{X}}, \\
& \operatorname{tr}_{X}^{R}(s)=e_{\bar{X}} \circ\left(\left(\alpha_{X} \circ s\right) \otimes \operatorname{id}_{\bar{X}}\right) \circ d_{X} .
\end{aligned}
$$

(Both traces take values in End 1.) The left and right dimensions of $X$ are defined by $d_{L / R}(X)=t r_{X}^{L / R}\left(\operatorname{id}_{X}\right)$.

The traces satisfy $\operatorname{tr}_{X}(s \circ t)=\operatorname{tr}_{Y}(t \circ s)$ for all $t: X \rightarrow Y$ and $s: Y \rightarrow X$, as well as $\operatorname{tr}_{X \otimes Y}(s \otimes t)=$ $\operatorname{tr}_{X}(s) \operatorname{tr}_{Y}(t)$ for $s \in$ End $X, t \in$ End $Y$. If $X$ is absolutely simple, the dimensions defined in terms of the traces are connected to the intrinsic squared dimension by $d_{L}(X) d_{R}(X)=d^{2}(X)$.

A pivotal category is called spherical [10] if $\operatorname{tr}_{X}^{L}=\operatorname{tr}_{X}^{R}$ for all $X \in \mathcal{C}$. In this case we have $d_{L}(X)=d_{R}(X)$ and simply write $d(X)$. In fact, a pivotal category that is semisimple over a field is spherical if and only if $d_{L}(X)=d_{R}(X)$ for every $X$. To this day, all known examples of pivotal fusion categories are spherical. (E.g., if $H$ is a finite dimensional semisimple Hopf algebra in characteristic zero, one automatically has $S^{2}=$ id and therefore sphericity of the module category.)

As noted before, in a hermitian category, duals (if they exist) are always two-sided. In a unitary category there is a canonical way of defining traces of endomorphisms, giving rise to positive dimensions, that makes no use of spherical structures, cf. [57]. But as shown by Yamagami [97, a unitary category always admits a unique spherical structure that gives rise to these traces. Note, however, there are $\mathbb{C}$-linear categories that do not admit a unitary structure, cf. [82. (Essentially, quantum group categories at odd root of unity.)

\section{$3.2 \quad$ Fusion categories}

Let $k$ be an algebraically closed field. A $k$-linear tensor category is called fusion category if it has finite dimensional hom-spaces, is semisimple with finitely many isomorphism classes of simple objects, the unit $\mathbf{1}$ is absolutely simple and all objects have duals (which are automatically two-sided by semisimplicity). A fusion subcategory of a fusion category is a full tensor subcategory that is again fusion. (I.e. closed under direct sums and duals.)

Even for fusion categories it is unknown whether pivotal structures always exist. (See 27] for work currently in progress.) But there is a result in this direction:

3.6 Theorem [32] Let $\mathcal{C}$ be a fusion category over $\mathbb{C}$ and let ${ }^{\vee}-$ be an arbitrary left dual structure. Then the tensor functor $\vee \vee \vee \vee \_$is naturally monoidally isomorphic to the identity functor. 
In a fusion category, we can find mutually non-isomorphic simple objects $X_{i}$, indexed by a finite set $I$, such that every object is a finite direct sum of the objects $X_{i}, i \in I$. There is a distinguished index $0 \in I$ such that $X_{0}=1$. Now we can define non-negative integers $N_{i j}^{k} \in \mathbb{N}_{0}$ via $X_{i} \otimes X_{j} \cong \oplus_{k \in I} N_{i j}^{k} X_{k}$. These numbers have various obvious properties, like $N_{i 0}^{j}=N_{0 i}^{j}=\delta_{i, j}$. We also have $N_{i j}^{0}=\delta_{i, \bar{\jmath}}$, where $i \mapsto \bar{\imath}$ is the involution on $I$ defined by $\overline{X_{i}} \cong X_{\bar{\imath}}$. The structure $\left(I, 0, i \mapsto \bar{\imath}, N_{. . .}\right)$is known as a discrete hypergroup. (In particular, every group $G$ gives rise to a hypergroup, taking $I=G, 0=e, \bar{g}=g^{-1}$ and $N_{g, h}^{k}=\delta_{g h, k}$.)

For every discrete hypergroup $(I, \cdots)$ there is a unique group $G(I)$ equipped with a map $\partial: I \rightarrow G(I)$ satisfying

$$
\partial 0=e, \quad \partial \bar{\imath}=(\partial i)^{-1}, \quad \text { and } \quad N_{i, j}^{k}>0 \Rightarrow \partial k=\partial i \cdot \partial j
$$

and being universal for such maps. (Thus for every map $\partial^{\prime}: I \rightarrow H$, where $H$ is a group, satisfying the same axioms as $\partial$ there is a unique group homomorphism $\alpha: G \rightarrow H$ such that $\partial^{\prime}=\alpha \circ \partial$.) In view of this property, $G(I)$ is called the (universal) grading group of the hypergroup $I$. (Also 'groupification' might be a good term, in analogy to abelianization.) Cf. 11, 42. Notice that $G(I)$ is abelian when $\mathcal{C}$ is braided. If $H$ is a compact group, let $\widehat{H}$ be the hypergroup corresponding to the semisimple category Rep $H$. Then the (discrete abelian) grading group $G(\widehat{H})$ is canonically isomorphic to Pontrjagin dual of the (compact abelian) center $Z(H)$ of $H$, cf. 69.

Each matrix $N_{i}=\left(N_{i j}^{k}\right)_{j, k}$ is irreducible and has non-negative entries. Thus it has a unique positive PerronFrobenius eigenvalue which is denoted as $\operatorname{FPdim}\left(X_{i}\right)$, the Frobenius-Perron dimension of $X_{i}$, cf. [32]. The Frobenius-Perron dimension [32] of the fusion category $\mathcal{C}$ is defined by

$$
\mathrm{FP} \operatorname{dim}(\mathcal{C})=\sum_{i \in I} \mathrm{FP} \operatorname{dim}\left(X_{i}\right)^{2}
$$

By definition, $\mathrm{FPdim}(X)$ and $\mathrm{FPdim}(\mathcal{C})$ live in $\mathbb{R}_{>0}$ rather than in the ground field $k$, but if $\mathbb{R} \subset k$ it makes sense to compare FPdim $(X)$ with $d^{2}(X)$ (which is canonically defined for all simple objects) and with $d(X)$ (if $\mathcal{C}$ has a spherical structure). It is classical that the FP-dimension is the unique positive dimension function on a finite hypergroup $I$. In particular the Frobenius-Perron dimension coincides with the positive dimension function defined on unitary categories with duals [57. (Recall that the latter arises from a unique spherical structure.) Somewhat more generally, if $\mathcal{C}$ is spherical over $k \supset \mathbb{R}$ and $d(X)>0$ for all $X$ then $d(X)=\mathrm{FP} \operatorname{dim}(X)$ for all $X$. Since not every fusion category over $\mathbb{C}$ is unitarizable (in the sense of admitting a positive $*$-operation) it is important that there are the following remarkable results:

\subsection{Theorem [32] Let $\mathcal{C}$ be a fusion category over $\mathbb{C}$. Then}

(i) For every simple $X$, one has $0<d^{2}(X) \leq \mathrm{FP} \operatorname{dim}(X)^{2}$. Thus $1 \leq \operatorname{dim} \mathcal{C} \leq \mathrm{FP} \operatorname{dim} \mathcal{C}$.

(ii) If $\operatorname{dim} \mathcal{C}=\mathrm{FP} \operatorname{dim}(\mathcal{C})$ (equivalent to $d^{2}(X)=\mathrm{FP} \operatorname{dim}(X)^{2}$ for every simple $X$ ) $\mathcal{C}$ admits a unique spherical structure for which $d(X)=\mathrm{FP} \operatorname{dim}(X)>0$ for every $X$. Such categories are called pseudo-unitary.

\subsection{Algebras in tensor categories and their modules}

A considerable part of (commutative) algebra can be generalized from the symmetric categories Ab and Vect $k$ to arbitrary (braided) tensor categories. This plays an important rôle in the structural study of such categories and in particular of braided fusion categories. In this subsection we discuss some facts that do not require a braiding.

3.8 Definition Let $\mathcal{C}$ be a strict tensor category. An algebra (or monoid) in $\mathcal{C}$ is a triple $(A, m, \eta)$, where $A \in \mathcal{C}, m: A \otimes A \rightarrow A$ and $\eta: \mathbf{1} \rightarrow A$ satisfy $m \circ\left(m \otimes \mathrm{id}_{A}\right)=m \circ\left(\operatorname{id}_{A} \otimes m\right)$ (associativity) and $m \circ\left(\eta \otimes \operatorname{id}_{A}\right)=$ $\operatorname{id}_{A}=m \circ\left(\mathrm{id}_{A} \otimes \eta\right)$ (unit property). If $\mathcal{C}$ is non-strict, one inserts associativity isomorphisms at the appropriate places.

3.9 REMARK At least in categories that are not linear over a field, it would be more appropriate to speak of monoids rather than algebras, but the latter term is used much more in the recent literature. 
3.10 Definition If $\mathcal{C}$ is a tensor category and $(A, m, \eta)$ is an algebra in $\mathcal{C}$ then a left $A$-module (in $\mathcal{C})$ is a pair $(X, \mu)$ where $X \in \mathcal{C}$ and $\mu: A \otimes X \rightarrow X$ satisfies $\mu \circ\left(m \otimes \operatorname{id}_{X}\right)=\mu \circ\left(\operatorname{id}_{A} \otimes \mu\right)$ and $\mu \circ\left(\eta \otimes \operatorname{id}_{X}\right)=\operatorname{id}_{X}$.
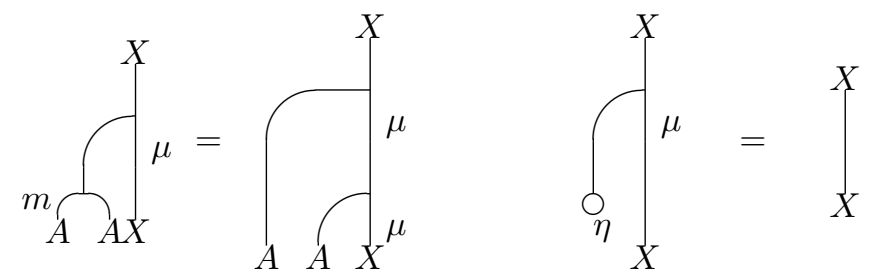

The left $A$-modules in $\mathcal{C}$ form a category, denoted $A$-Mod $\operatorname{Mor}_{A} \mathcal{C}$, with hom-sets

$$
\operatorname{Hom}_{A} \mathcal{C}\left((X, \mu),\left(X^{\prime}, \mu^{\prime}\right)\right)=\left\{t \in \operatorname{Hom}_{\mathcal{C}}\left(X, X^{\prime}\right) \mid t \circ \mu=\mu^{\prime} \circ\left(\operatorname{id}_{A} \otimes t\right)\right\} .
$$

Right modules and bimodules are defined analogously.

There is a functor $F_{A}: \mathcal{C} \rightarrow{ }_{A} \mathcal{C}$, the free module functor, defined by $F_{A}: X \mapsto\left(A \otimes X, m \otimes \operatorname{id}_{X}\right)$. Notice that for every $(X, \mu) \in{ }_{A} \mathcal{C}$ we have $\mu \in \operatorname{Hom}_{A} \mathcal{C}(F(X),(X, \mu))$. The functor $F_{A}$ is faithful provided $s \mapsto \operatorname{id}_{A} \otimes s$ is injective, which usually is the case. Since the maps

$$
\begin{array}{ll}
\operatorname{Hom}_{A} \mathcal{C}\left(F_{A}(X), F_{A}(Y)\right) \rightarrow \operatorname{Hom}_{\mathcal{C}}(A \otimes X, Y), & s \mapsto s \circ\left(\eta \otimes \operatorname{id}_{Y}\right), \\
\operatorname{Hom}_{\mathcal{C}}(A \otimes X, Y) \rightarrow \operatorname{Hom}_{A} \mathcal{C}\left(F_{A}(X), F_{A}(Y)\right), & t \mapsto\left(m \otimes \operatorname{id}_{Y}\right) \circ\left(\operatorname{id}_{A} \otimes t\right)
\end{array}
$$

are inverses of each other, we have a bijection $\operatorname{Hom}_{A} \mathcal{C}\left(F_{A}(X), F_{A}(Y)\right) \cong \operatorname{Hom}_{\mathcal{C}}(A \otimes X, Y)$. Thus $F_{A}$ will in general not be full, and it can happen that $F_{A}$ trivializes an object $X \in \mathcal{C}$ in the sense of mapping it to a multiple of the unit object $(A, m)$ of ${ }_{A} \mathcal{C}$.

The free $A$-modules form a full subcategory of ${ }_{A} \mathcal{C}$, but in order to say more about the module category, one needs a descent-type assumption like the following:

3.11 Definition An algebra $(A, m, \eta)$ in a tensor category is called separable if the multiplication morphism admits a splitting that is a morphism of $A$ - $A$ bimodules, i.e. a morphism $\widetilde{m}: A \rightarrow A \otimes A$ satisfying

$$
\left(m \otimes \mathrm{id}_{A}\right) \circ\left(\mathrm{id}_{A} \otimes \tilde{m}\right)=\tilde{m} \circ m=\left(\mathrm{id}_{A} \otimes m\right) \circ\left(\tilde{m} \otimes \mathrm{id}_{A}\right), \quad m \circ \tilde{m}=\mathrm{id}_{A} .
$$

If $\mathcal{C}$ is $k$-linear and $\operatorname{dim} \operatorname{Hom}(\mathbf{1}, A)=1$ then the algebra $(A, m, \eta)$ is called connected.

3.12 Lemma If $\mathcal{C}$ is a tensor category and $(A, m, \eta)$ a separable algebra, then every module $(X, \mu) \in{ }_{A} \mathcal{C}$ is a quotient of the free module $F(X)$.

Proof. Let $\widetilde{m}: A \rightarrow A \otimes A$ be a splitting of $m$. Defining

$$
\gamma=\left(\operatorname{id}_{A} \otimes \mu\right) \circ\left(\widetilde{m} \otimes \operatorname{id}_{X}\right) \circ\left(\eta \otimes \operatorname{id}_{X}\right): X \rightarrow A \otimes X,
$$

an easy computation shows that $\gamma \in \operatorname{Hom}_{A} \mathcal{C}((X, \mu), F(X))$ and $\mu \circ \gamma=\operatorname{id}_{X}$. Thus $\mu \in \operatorname{Hom}_{A} \mathcal{C}(F(X),(X, \mu))$ is a split epimorphism.

3.13 Remark A notion similar to separability appeared in [18, where a morphism $\beta: 1 \rightarrow A \otimes A$ was required, satisfying axioms following from the above ones if one takes $\beta=\widetilde{m} \circ \eta$. (Notice that $\beta$ is what is actually used in the Lemma.) A related concept is that of a special Frobenius algebra, which is a quintuple $(A, m, \eta, \widetilde{m}, \varepsilon)$ where $(A, m, \eta, \widetilde{m})$ is a separable algebra and $(A, \widetilde{m}, \varepsilon)$ is a coalgebra. (In particular, $\widetilde{m}$ must be coassociative.)

3.14 Lemma [77, 32, 23] Let $\mathcal{C}$ be a fusion category and $(A, m, \eta)$ an algebra in $\mathcal{C}$. Then the following are equivalent:

(i) $A$ is separable.

(ii) The category ${ }_{A} \mathcal{C}$ is semisimple. 
(iii) The category $\mathcal{C}_{A}$ is semisimple.

(iv) The category ${ }_{A} \mathcal{C}_{A}$ is semisimple.

3.15 Remark For $\mathcal{C}$ spherical, (i) $\Rightarrow$ (iv) was already shown in [65], as part of the following.

3.16 TheOREM 65. If $\mathcal{C}$ is a spherical fusion category and $A \in \mathcal{C}$ a separable and connected algebra then $\mathcal{D}={ }_{A} \mathcal{C}_{A}$ is a spherical fusion category and it contains a separable connected algebra $B$ such that $\mathcal{C} \simeq{ }_{B} \mathcal{D}_{B}$. Calling fusion categories that are related in this way weakly monoidally Morita equivalent, weak monoidal Morita equivalence is an equivalence relation, denoted by $\approx$. If $\mathcal{C} \approx \mathcal{D}$ then $\operatorname{dim} \mathcal{C}=\operatorname{dim} \mathcal{D}$.

3.17 REMARK 1. If $H$ is a finite dimensional semisimple and co-semisimple Hopf algebra then $H-\operatorname{Mod} \approx$ $\widehat{H}-$ Mod.

2. The results of the theorem have been generalized to fusion categories in [32]. In that generality, one must use FPdim instead of dim.

\subsection{Module categories and categories of modules}

3.18 Definition If $\mathcal{M}$ is a category, End $\mathcal{M}$ denotes the category whose objects are the endofunctors of $\mathcal{M}$, i.e. functors $\mathcal{M} \rightarrow \mathcal{M}$, and whose morphisms are the natural transformations of these endofunctors. With $\otimes$ defined as composition of functors, End $\mathcal{M}$ is a strict tensor category. An alternative name for End $\mathcal{M}$ is $Z_{0}(\mathcal{M})$, the monoidal center of $\mathcal{M}$.

3.19 Definition Let $\mathcal{C}$ be a tensor category and $\mathcal{M}$ a category. A left, resp. right, $\mathcal{C}$-module structure on $\mathcal{M}$ is a tensor functor $\mathcal{C} \rightarrow$ End $\mathcal{M}$, resp. $\mathcal{C}^{\text {rev }} \rightarrow \operatorname{End} \mathcal{M}$. A left (right) $\mathcal{C}$-module category is a category $\mathcal{M}$ together with a left (right) $\mathcal{C}$-module structure. A module category is indecomposable if it is not the direct sum of two non-trivial module categories.

Left (right) $\mathcal{C}$-module categories form a 2-category, whose 1-morphisms are functors between module categories intertwining the $\mathcal{C}$-actions and whose 2-morphisms are natural transformations between module functors.

3.20 REMARK Unpacking the above definition, one finds that an action of a tensor category $\mathcal{C}$ on a category $\mathcal{M}$ is the same as a functor $\mathcal{C} \times \mathcal{M} \rightarrow \mathcal{M}$ satisfying certain properties like the existence of natural isomorphisms $(C \otimes D) \otimes M \rightarrow C \otimes(D \otimes M)$, etc. For such an approach, cf. e.g. [77].

If $\mathcal{C}$ is a tensor category and $(A, m, \eta)$ an algebra in $\mathcal{C}$ then the left module category ${ }_{A} \mathcal{C}$ has a structure as a right $\mathcal{C}$-module category: $(X, \mu) \otimes Y=\left(X \otimes Y, \mu \otimes \operatorname{id}_{Y}\right)$. Similarly, $\mathcal{C}_{A}$ has a left $\mathcal{C}$-module structure. The question now arises whether every $\mathcal{C}$-module category is of this form. For fusion categories this is the case:

3.21 TheOREm 77 Let $\mathcal{C}$ be a fusion category and $\mathcal{M}$ an indecomposable semisimple left module category over $\mathcal{C}$. Then there is a connected separable algebra $(A, m, \eta)$ in $\mathcal{C}$ and an equivalence $\mathcal{M} \rightarrow \mathcal{C}_{A}$ of module categories.

Thus (indecomposable, semisimple) module categories over a fusion category $\mathcal{C}$ and categories of modules for a separable algebra in $\mathcal{C}$ are essentially the same thing. This allows to discuss the weak monoidal Morita equivalence of 65] in terms of module categories: If $\mathcal{M}$ is a good module category over the fusion category $\mathcal{C}$ then the category $\operatorname{Hom}_{\mathcal{C}}(\mathcal{M}, \mathcal{M})$ of $\mathcal{C}$-module functors, which clearly is monoidal, actually is fusion and is called dual to $\mathcal{C}$ with respect to $\mathcal{M}$, cf. 32]. The connection with 65] is as follows: When $\mathcal{M}={ }_{A} \mathcal{C}$ then $\operatorname{Hom}_{\mathcal{C}}(\mathcal{M}, \mathcal{M}) \simeq{ }_{A} \mathcal{C}_{A}$, where ${ }_{A} \mathcal{C}_{A}$ acts on ${ }_{A} \mathcal{C}$ by tensoring from the left.

\section{Braided tensor categories}

\subsection{Centralizers in braided categories and the symmetric center $Z_{2}$}

In this section, we quickly discuss some aspects of braided tensor categories that do not require additional axioms like existence of duals or linearity over a ring or field. A braided tensor category is a tensor category $\mathcal{C}$ equipped with a braiding $c$. For the definition of the latter, we refer to Majid's contribution to the present volume. In principle, a braided tensor category should be written as $(\mathcal{C}, c)$, where $\mathcal{C}$ is a tensor category and $c$ a braiding on $\mathcal{C}$, but we will suppress the $c$. (After all, one usually does the same with the various items of the monoidal structure.) 
4.1 Definition Let $\mathcal{C}$ be a braided tensor category with braiding $c$. The opposite braiding $\widetilde{c}$ is defined by $\widetilde{c}_{X, Y}=\left(c_{Y, X}\right)^{-1}$. The tensor category $\mathcal{C}$ equipped with the braiding $\widetilde{c}$ is denoted $\widetilde{\mathcal{C}}$. by

In the graphical calculus (where we draw morphisms going upwards) the braidings $c$ and $\widetilde{c}$ are represented
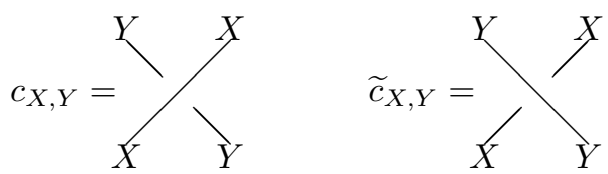

which is consistent in view of the isotopy

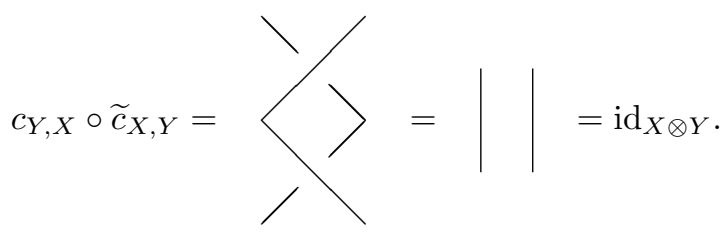

4.2 Remark 1. Clearly $\widetilde{\widetilde{c}}=c$ always holds. The stronger statement $\widetilde{c}=c$ is equivalent to

$$
c_{X, Y} \circ c_{Y, X}=\operatorname{id}_{Y \otimes X} \quad \forall X, Y \in \mathcal{C} .
$$

Recall that a BTC satisfying this additional condition is called a symmetric tensor category (STC). For this reason the braiding (=symmetry) of an STC is depicted by a crossing with unbroken lines. Conversely, the definition of BTCs is obtained from the usual definition of symmetric tensor categories by dropping the condition (4.1) that the braiding be involutive. (In doing so, one must add a second hexagon axiom which follows from the first in the presence of (4.1).)

2. Since examples of STCs abound, e.g. the category of sets, categories of modules and vector spaces, representation categories of groups, categories of sheaves, etc., it is quite astonishing that they were formalized only in 1963, cf. [14, 58. Considering that it is much harder to find interesting examples of non-symmetric BTCs, it is less surprising that their formalization took place only around 1985/6 in the first preprint versions of [48. To some extent, this was inspired by the surge of activity around new 'quantum' invariants in low dimensional topology [46, 39], quantum groups [28, conformal field theory [62] and algebraic quantum field theory [35] in the second half of the 1980s. But there are also considerations intrinsic to (higher) category theory as well as in 'old-fashioned' (non-'quantum') algebraic topology that lead to BTCs, cf. [48].

3. In a BTC, any two objects commute up to isomorphism: $X \otimes Y \cong Y \otimes X$. Looking for a stronger statement to be attached to the expression ' $X$ and $Y$ commute', one could think of $c_{X . Y}=\operatorname{id}_{X \otimes Y}$ or the slightly weaker $X \otimes Y=Y \otimes X$. But these are hardly ever satisfied in interesting BTCs. This essentially leaves us with only the following option.

\subsection{Definition Let $\mathcal{C}$ be a $B T C$.}

(a) Two objects $X, Y \in \mathcal{C}$ are said to commute if $c_{X, Y}=\widetilde{c}_{X, Y}$, equivalently $c_{X, Y} \circ c_{Y, X}=\mathrm{id}_{Y \otimes X}$.

(b) Let $\mathcal{D} \subset \mathcal{C}$ be a subcategory. The centralizer $C_{\mathcal{C}}(\mathcal{D})$ is the full subcategory of $\mathcal{C}$ defined by

$$
\operatorname{Obj} C_{\mathcal{C}}(\mathcal{D})=\left\{X \in \mathcal{C} \mid c_{X, Y} \circ c_{Y, X}=\operatorname{id}_{Y \otimes X} \forall Y \in \mathcal{D}\right\} .
$$

(c) The symmetric center $Z_{2}(\mathcal{C})$ is defined as $C_{\mathcal{C}}(\mathcal{C})$, i.e. the full subcategory defined by

$$
\operatorname{Obj} Z_{2}(\mathcal{C})=\left\{X \in \mathcal{C} \mid c_{X, Y} \circ c_{Y, X}=\operatorname{id}_{Y \otimes X} \forall Y \in \mathcal{C}\right\} .
$$

4.4 REMARK 1. The definition of $Z_{2}(\mathcal{C})$ is due to [18, 64, but the concept first appeared ten years earlier in the context of algebraic quantum field theory [80. The centralizer $C_{\mathcal{C}}(\mathcal{D})$ seems first to have appeared in 67 .

2. In most of the literature, $\mathcal{D}^{\prime}$ is written instead of $C_{\mathcal{C}}(\mathcal{D})$. When the ambient category $\mathcal{C}$ is fixed, there is no risk of confusion.

3. $C_{\mathcal{C}}(\mathcal{D})$ depends only on the objects of $\mathcal{D}$. One easily sees, for any $\mathcal{D}$, that the monoidal unit 1 lies in $C_{\mathcal{C}}(\mathcal{D})$ and that $C_{\mathcal{C}}(\mathcal{D})$ is closed under tensor products, thus is is a full monoidal subcategory. Furthermore, it is closed under isomorphisms (i.e. replete) and under direct sums, if they exist. The braiding that $C_{\mathcal{C}}(\mathcal{D})$ inherits from $\mathcal{C}$ in fact is a symmetry when $\mathcal{D}=\mathcal{C}$, thus $Z_{2}(\mathcal{C})$ is an STC. In fact, a BTC $\mathcal{C}$ is symmetric if and only if $\mathcal{C}=Z_{2}(\mathcal{C})$. 
4. The objects of $Z_{2}(\mathcal{C})$ are called transparent [18], since for braidings involving them it there is no difference between over- and under-crossings, or central.

5. The name 'center' for $Z_{2}(\mathcal{C})$ is amply justified by considerations from higher category theory, cf. e.g. 3, 4, some of which already played a rôle in [48. In higher category theory, an infinite family of center constructions is considered. In the more limited context of 1-categories, one deals with the bicategories consisting of categories, tensor categories, braided tensor categories, symmetric tensor categories, respectively. Notice that moving rightwards in this list adds a piece of structure (tensor structure, braiding) except in the last step, where a condition is added, to wit (4.1). It is clear that there are forgetful (2-)functors moving leftwards in this list. More interestingly, there are constructions, called centers, in the opposite direction. We have just defined the symmetric center $Z_{2}(\mathcal{C})$ of a BTC $\mathcal{C}$, and the monoidal center $Z_{0}(\mathcal{C})=$ End $\mathcal{C}$ of a category $\mathcal{C}$ was given in Definition 3.18. The braided center $Z_{1}(\mathcal{C})$ of a tensor category $\mathcal{C}$ will be defined below. (These constructions are categorifications of their simpler analogues for 0 -categories, i.e. sets, where we deal with the 1-categories of sets, monoids and commutative monoids. The center $Z_{1}(M)$ of a monoid is well known, whereas the center $Z_{0}(S)$ of a set $S$ is the monoid of endomaps of $S$.)

Notice that the center constructions are compatible with equivalences of categories, but not with more general functors. Thus they are functorial only on the sub-bicategories of CAT, $\otimes$-CAT, etc., whose 1-morphisms are equivalences of categories, tensor categories (TCs), etc.

6. In view of the above, one realizes that symmetric tensor categories, which play a rather prominent rôle in large parts of mathematics, are but one extreme case of braided tensor categories, singled out by the condition that they coincide with their symmetric centers. This makes it natural ask whether interesting things can be said in the opposite extreme case, namely when the center of a BTC $\mathcal{C}$ is trivial in the sense of containing only what it must contain, to wit the unit object and its direct sums. (Compare with the theory of von Neumann algebras, where the commutative ones and those with trivial center ('factors'), play distinguished rôles.) This is indeed the case, modular categories just being braided categories having a bit more structure and having trivial symmetric center $Z_{2}(\mathcal{C})$. Since this section is devoted to results requiring no additional axioms, the study of modular categories will begin later.

\subsection{Rambling remarks on the construction of proper BTCs}

So far, we have not given any example of a non-symmetric BTC. The simplest one, the free braided tensor category $\mathcal{B}$ generated by one object, or just the braid category, is constructed from the braid groups $B_{n}$ and could have been found long before [48. Its objects are the non-negative integers $\{0,1,2, \ldots\}$ with addition as tensor product. The category is discrete, i.e. $\operatorname{Hom}_{\mathcal{B}}(n, m)=\emptyset$ when $n \neq m$, the endomorphisms given by $\operatorname{End}_{\mathcal{B}}(n)=B_{n}$ with composition as in $B_{n}$ (i.e. concatenation of braids). The tensor product of morphisms is given by horizontal juxtaposition of braids, and the braiding is defined as in

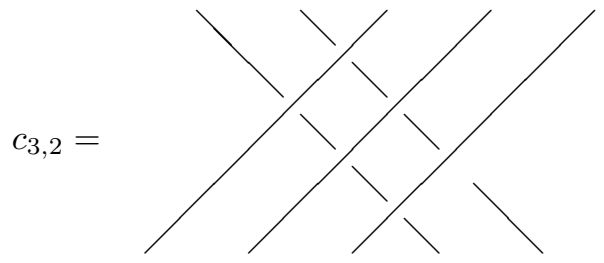

This category clearly is not symmetric. (For example, under the isomorphism $B_{2} \rightarrow \mathbb{Z}$, the braid $c_{1,1} \circ c_{1,1}$ is mapped to $2 \neq 0$.)

An attempt to systematize the known constructions of braided categories was made in [72, where three types of constructions were distinguished:

1. Braided deformations of symmetric tensor categories.

2. Free (=topological) constructions.

3. The braided center $Z_{1}(\mathcal{C})$ of a tensor category.

While the philosophies behind these three approaches are quite different, they are by no means mutually exclusive. In fact, the most interesting braided categories, to wit the representation categories of quantum groups, can be understood in terms of all three constructions! 
Space constraints do not allow to say much about the deformation approach. While it is usually formulated in terms of a ' $q$-deformation' of the universal enveloping algebra $U(\mathfrak{g})$ of a simple Lie algebra, giving rise to a quasi-triangular Hopf algebra $U_{q}(\mathfrak{g})$, cf. e.g. [61, 49, 21, one may argue that (as always?) a categorical perspective provides additional insight. Namely, the representation categories $\mathcal{C}(\mathfrak{g}, q)=U_{q}(\mathfrak{g})-\operatorname{Mod}$ can be obtained directly by deformation of the STCs $\mathcal{C}(\mathfrak{g})=U(\mathfrak{g})-$ Mod. Such deformations are controlled by the third Davydov-Yetter cohomology, cf. 22, 99, 100, and one can show 32] that $H^{3}(D(\mathfrak{g})-\mathrm{Mod})$ is one-dimensional for a simple Lie algebra $\mathfrak{g}$, explaining the one-parameter family of $q$-deformations. Actually constructing the braided deformed category $\mathcal{C}(\mathfrak{g}, q)$ from $\mathcal{C}(\mathfrak{g})$ can be done formally using Drinfeld associators [50] or analytically (i.e. non-formally) using the Knizhnik-Zamolodchikov connection [52]. The categories thus obtained can be shown to be equivalent to the representation categories of the corresponding quantum groups.

The approach via 'free constructions' generalizes the construction of the braid category $\mathcal{B}$ given above. Given a tensor category $\mathcal{C}$, there is a free braided tensor category $F \mathcal{C}$ over $\mathcal{C}$, which reduces to $\mathcal{B}$ when $\mathcal{C}$ is the trivial tensor category $\{\mathbf{1}\}$, cf. [48. This construction provides a left adjoint to the forgetful 2 -functor from the bicategory of braided tensor categories to the bicategory of tensor categories. There are analogous versions of this construction in case one studies categories with additional structures, like duals or linearity over a field. Most important, at least as far as connections with low dimensional topology are concerned, are the the various categories of tangles, which can be considered as free rigid braided category, free ribbon category, etc., generated by one object, cf. [85, 37, 87, 49, 100. The tangle categories are not linear over a field, but can easily be linearized using the the free vector space functor from sets to vector spaces. The categories thus obtained are still too generic and too big (in the sense of having infinite dimensional hom-spaces) to be really interesting, but quotienting them by a suitable ideal defined, e.g., in terms of a link invariant, one can obtain rigid braided or ribbon categories with finite dimensional hom-spaces. For appropriate choices of the link invariant (HOMFLY or Kauffman polynomials), one actually obtains the representation categories of the quantum groups of types A-D, cf. [91, 12, 13, 16, including the most interesting (and difficult) root-of-unity case. Again, there is no space to go into this any further.

However, beginning in the next subsection, we will have more to say about the third approach to the construction of modular categories, the braided center construction, since it is of considerable relevance for the structure theory of modular categories. This construction will play a central rôle in most of what follows.

\subsection{The braided center $Z_{1}(\mathcal{C})$}

For simplicity, we give the following definition only for strict tensor categories, but the generalization is straightforward.

\subsection{Definition/Proposition Let $\mathcal{C}$ be a strict tensor category.}

(a) Let $X \in \mathcal{C}$. A half braiding $e_{X}$ for $X$ is a family $\left\{e_{X}(Y): X \otimes Y \stackrel{\cong}{\longrightarrow} Y \otimes X\right\}_{Y \in \mathcal{C}}$ of isomorphisms, natural w.r.t. $Y$, satisfying $e_{X}(\mathbf{1})=\mathrm{id}_{X}$ and

$$
e_{X}(Y \otimes Z)=\operatorname{id}_{Y} \otimes e_{X}(Z) \circ e_{X}(Y) \otimes \operatorname{id}_{Z} \quad \forall Y, Z \in \mathcal{C} .
$$

(b) Let $Z_{1}(\mathcal{C})$ be the category whose objects are pairs $\left(X, e_{X}\right)$ consisting of an object and a half-braiding, the hom-sets being given by

$$
\operatorname{Hom}_{Z_{1}(\mathcal{C})}\left(\left(X, e_{X}\right),\left(Y, e_{Y}\right)\right)=\left\{t \in \operatorname{Hom}_{\mathcal{C}}(X, Y) \mid \operatorname{id}_{X} \otimes t \circ e_{X}(Z)=e_{Y}(Z) \circ t \otimes \operatorname{id}_{X} \quad \forall Z \in \mathcal{C}\right\} .
$$

Now a tensor product of two objects is defined by $\left(X, e_{X}\right) \otimes\left(Y, e_{Y}\right)=\left(X \otimes Y, e_{X \otimes Y}\right)$, where

$$
e_{X \otimes Y}(Z)=e_{X}(Z) \otimes \operatorname{id}_{Y} \circ \operatorname{id}_{X} \otimes e_{Y}(Z) .
$$

The tensor unit is $\left(\mathbf{1}, e_{\mathbf{1}}\right)$ where $e_{\mathbf{1}}(X)=\mathrm{id}_{X}$. Defining composition and tensor product of morphisms to be inherited from $\mathcal{C}$, one verifies that $Z_{1}(\mathcal{C})$ is a strict tensor category. Finally,

$$
c_{\left(X, e_{X}\right),\left(Y, e_{Y}\right)}=e_{X}(Y)
$$

defines a braiding. The braided tensor category $Z_{1}(\mathcal{C})$ will be called the braided center of $\mathcal{C}$.

4.6 REMARK 1 . Usually, $Z_{1}(\mathcal{C})$ is denoted by $Z(\mathcal{C})$. We wrote $Z_{1}$ to avoid confusion with the symmetric center $Z_{2}$ (if $\mathcal{C}$ is braided, both $Z_{1}(\mathcal{C})$ and $Z_{2}(\mathcal{C})$ are defined) but later we will drop the subscript and identify $Z=Z_{1}$. 
2. In the same way as $Z_{2}(\mathcal{C})=C_{\mathcal{C}}(\mathcal{C})$ is a special case of the centralizer $C_{\mathcal{C}}(\mathcal{D})$, there is a 'relative center' $Z_{1}(\mathcal{C}, \mathcal{D})$ for a pair $\mathcal{D} \subset \mathcal{C}$. Its objects are pairs $\left(X, e_{X}\right)$ where $X \in \mathcal{C}$ and $e_{X}$ is a family of isomorphisms $X \otimes Y \rightarrow Y \otimes X$ for all $Y \in \mathcal{D} . \quad Z_{1}(\mathcal{C}, \mathcal{D})$ is always monoidal, but not necessarily braided, and we have $Z_{1}(\mathcal{C}, \mathcal{C})=Z_{1}(\mathcal{C})$

3. The definition of $Z_{1}$ appeared in [47] and [60. The second reference also gave $Z(\mathcal{C}, \mathcal{D})$ and attributed $Z_{1}(\mathcal{C})$ to unpublished work of Drinfeld, which led many authors to call $Z_{1}(\mathcal{C})$ the 'Drinfeld center'.

4. Despite its being somewhat involved, the definition of $Z_{1}(\mathcal{C})$ is quite natural. It is nevertheless instructive to give an interpretation in terms of bicategories. The point is that a (strict) tensor category is 'the same' as a (strict) 2-category with one object, and similarly, braided tensor categories correspond to monoidal 2-categories with one object 48 . Now, let $\mathcal{E}$ be the 2 -category with one object corresponding to the tensor category $\mathcal{C}$ and let $\mathcal{F}$ be the monoidal 2-category $Z_{0}(\mathcal{E})$ of endo-2-functors of $\mathcal{E}$. If $\mathcal{F}_{1} \subset \mathcal{F}$ is the full sub-2-category retaining only the unit object $\mathbf{1}=\mathrm{id}_{\mathcal{E}}$, it turns out that the braided category corresponding to $\mathcal{F}_{1}$ is nothing but $Z_{1}(\mathcal{C})$.

5 . The construction of $Z_{1}(\mathcal{C})$ was preceded and probably motivated by Drinfeld's definition of the quantum double $D(H)$, which is a quasi-triangular Hopf algebra, of a Hopf algebra $H$. The two constructions are closely related, for one can construct, at least if $H$ is finite dimensional, an equivalence

$$
D(H)-\operatorname{Mod} \simeq Z_{1}(H-\operatorname{Mod})
$$

of braided tensor categories. Cf. [49]. Since it is not true that all tensor categories arise from Hopf algebras, the construction of the braided center $Z_{1}(\mathcal{C})$ can be considered a generalization of the Hopf algebraic quantum double. (One might also find the definition of $Z_{1}(\mathcal{C})$ more natural than that of $D(H)$.)

6 . If $\mathcal{C}$ is $k$-linear, spherical or a $*$-category (=unitary category), the same holds for $Z_{1}(\mathcal{C})$. Other properties are much harder to show. In situations where a tensor category $\mathcal{C}$ is not of the form $H-$ Mod for some Hopf algebra $H$, it can actually be quite difficult to construct objects of $Z_{1}(\mathcal{C})$ different from $\mathbf{1}_{Z_{1}(\mathcal{C})}$. There are situations where $\mathcal{C}$ is quite big, but $Z_{1}(\mathcal{C})$ is 'trivial' (in the sense that $X \cong \mathbf{1} \forall X$ ): This happens if $\mathcal{C}_{0}$ is a category and $\mathcal{C}_{1}=Z_{0}\left(\mathcal{C}_{0}\right)=\operatorname{End}\left(\mathcal{C}_{0}\right)$, categorifying the simple fact that the center (in the usual sense) $Z_{1}(M)$ of the monoid $M=Z_{0}(S)$ of endomaps of any set $S$ is trivial in the sense of $Z_{1}\left(Z_{0}(S)\right)=\left\{\operatorname{id}_{S}\right\}$.

As we will see later, the situation is much better if $\mathcal{C}$ is a fusion category.

In view of its construction, it is clear that there is a forgetful tensor functor

$$
K: Z_{1}(\mathcal{C}) \rightarrow \mathcal{C}, \quad\left(X, e_{X}\right) \mapsto X .
$$

In general, there is no natural functor, in particular not an inclusion, from $\mathcal{C}$ to $Z_{1}(\mathcal{C})$. There are two exceptions: If $\mathcal{C}$ is a fusion category, $K$ has a 2 -sided (non-monoidal) adjoint $I: \mathcal{C} \rightarrow Z_{1}(\mathcal{C})$. This will be discussed later.

The other exception is the case where $\mathcal{C}$ comes with a braiding $c$. While the definition of $Z_{1}(\mathcal{C})$ makes no reference to $c$, its existence has many consequences, cf. [66]:

1. There are two tensor functors $F_{1}, F_{2}: \mathcal{C} \rightarrow Z_{1}(\mathcal{C})$, given by

$$
F_{1}(X)=\left(X, e_{X}\right) \text { with } e_{X}(Y)=c_{X, Y}, \quad F_{2}(X)=\left(X, \tilde{e}_{X}\right) \text { with } \tilde{e}_{X}(Y)=\tilde{c}_{X, Y}
$$

Both functors are braided tensor functors from $\mathcal{C}=(\mathcal{C}, c)$ and $\widetilde{\mathcal{C}}=(\mathcal{C}, \widetilde{c})$, respectively, to $Z_{1}(\mathcal{C})$.

2. $F_{1}$ and $F_{2}$ are full and faithful, i.e. give embeddings $\mathcal{C} \hookrightarrow Z_{1}(\mathcal{C}), \widetilde{\mathcal{C}} \hookrightarrow Z_{1}(\mathcal{C})$. E.g.,

$$
\begin{aligned}
& \operatorname{Hom}_{Z_{1}(\mathcal{C})}\left(F_{1}(X), F_{1}(Y)\right)=\operatorname{Hom}_{Z_{1}(\mathcal{C})}\left(\left(X, c_{X, \bullet}\right),\left(Y, c_{Y, \bullet}\right)\right) \\
& \quad=\left\{t \in \operatorname{Hom}_{\mathcal{C}}(X, Y) \mid \operatorname{id}_{X} \otimes t \circ c_{X, Z}=c_{Y, Z} \circ t \otimes \operatorname{id}_{X} \quad \forall Z \in \mathcal{C}\right\}=\operatorname{Hom}_{\mathcal{C}}(X, Y),
\end{aligned}
$$

due to the naturality of $c$ w.r.t. both arguments.

3. The full subcategories $F_{1}(\mathcal{C}) \subset Z_{1}(\mathcal{C})$ and $F_{2}(\widetilde{\mathcal{C}}) \subset Z_{1}(\mathcal{C})$ commute with each other: By the definitions of $Z_{1}(\mathcal{C})$ and of $F_{1}, F_{2}$, we have

$$
c_{F_{1}(X), F_{2}(Y)} \circ c_{F_{2}(Y), F_{1}(X)}=c_{X, Y} \circ \widetilde{c}_{Y, X}=c_{X, Y} \circ c_{X, Y}^{-1}=\operatorname{id}_{Y \otimes X} .
$$

An almost equally simple argument shows that $F_{1}(\mathcal{C}), F_{2}(\widetilde{\mathcal{C}})$ are each others centralizers:

$$
F_{1}(\mathcal{C})^{\prime}=C_{Z_{1}(\mathcal{C})}\left(F_{1}(\mathcal{C})\right)=F_{2}(\widetilde{\mathcal{C}}), \quad F_{2}(\widetilde{\mathcal{C}})^{\prime}=C_{Z_{1}(\mathcal{C})}\left(F_{2}(\widetilde{\mathcal{C}})\right)=F_{1}(\mathcal{C})
$$

Consequentially,

$$
F_{1}(\mathcal{C})^{\prime \prime}=F_{1}(\mathcal{C}), \quad F_{2}(\widetilde{\mathcal{C}})^{\prime \prime}=F_{2}(\widetilde{\mathcal{C}}) .
$$

This is nice since a priori we only know that $\mathcal{D} \subset \mathcal{D}^{\prime \prime}$ for a tensor subcategory $\mathcal{D} \subset \mathcal{C}$. Again, for a fusion category $\mathcal{C}$, one can prove much stronger results. 
4. Since $F_{1}(\mathcal{C})$ and $F_{2}(\widetilde{\mathcal{C}})$ are full subcategories of $Z_{1}(\mathcal{C})$, so is their intersection, and one finds

$$
F_{1}(\mathcal{C}) \cap F_{2}(\widetilde{\mathcal{C}})=F_{1}\left(Z_{2}(\mathcal{C})\right)=F_{2}\left(Z_{2}(\mathcal{C})\right)
$$

5. In view of item $4, F_{1}$ and $F_{2}$ combine to a braided tensor functor

$$
H: \mathcal{C} \times \widetilde{\mathcal{C}} \rightarrow Z_{1}(\mathcal{C}), \quad(X, Y) \mapsto F_{1}(X) \otimes F_{2}(Y) .
$$

In view of 5 ., this functor will be neither full nor faithful in general: If $X \in Z_{2}(\mathcal{C})$ and $X \neq \mathbf{1}$ then $(X, \mathbf{1}) \varsubsetneqq(\mathbf{1}, X)$ but $H((X, \mathbf{1}))=F_{1}(X)=F_{2}(X)=H((\mathbf{1}, X))$, thus $H$ does not reflect isomorphisms. However, we will see that the linearized version $\mathcal{C} \otimes \widetilde{\mathcal{C}} \rightarrow Z_{1}(\mathcal{C})$ of $H$ actually is an equivalence when $\mathcal{C}$ is modular.

Another definition, due to [15], involving the braided center will be useful later:

4.7 Definition Let $\mathcal{C}$ be a $B T C, \mathcal{D}$ a tensor category and $F: \mathcal{C} \rightarrow \mathcal{D}$ a tensor functor. $A$ central structure on $F$ is a braided tensor functor $\widehat{F}: \mathcal{C} \rightarrow Z_{1}(\mathcal{D})$ such that $K \circ \widehat{F}=F$. Here $K: Z_{1}(\mathcal{D}) \rightarrow \mathcal{D}$ is the tensor functor that forgets the half-braiding, thus $\widehat{F}$ is a lift of $F$ from $\mathcal{C}$ to $Z_{1}(\mathcal{C})$.

4.8 Remark From the point of view of the Baez-Dolan picture of ' $k$-tuply monoidal $n$-categories', cf. [3], it is interesting to note the close analogy between central functors $\widehat{F}: \mathcal{C} \rightarrow Z_{1}(\mathcal{D}$ ) (where $\mathcal{C}$ is braided and $\mathcal{D}$ just monoidal) and actions $F: \mathcal{C} \rightarrow Z_{0}(\mathcal{D})$ (with $\mathcal{D}$ a category and $\mathcal{C}$ monoidal). In both cases, the center $Z_{0}$ resp. $Z_{1}$ on the r.h.s. serves to create the piece of structure (monoidal, braiding) that is needed in order to talk about a monoidal functor $F$ or braided functor $\widehat{F}$.

\subsection{Algebras and modules in BTCs and module categories of BTCs}

4.9 Definition Let $\mathcal{C}$ be a braided tensor category. An algebra $(A, m, \eta)$ in $\mathcal{C}$ is called commutative if $m \circ c_{A, A}=$ m. A commutative separable algebra is called étale.

In the case where $\mathcal{C}$ is braided and the algebra $(A, m, \eta)$ in $\mathcal{C}$ is commutative, we would like ${ }_{A} \mathcal{C}$ to be a (braided) tensor category. In order for this to hold we need an additional assumption:

From now on, we require without further mention that $\mathcal{C}$ has coequalizers. (Cf. [59] for the definition.)

Later on, all categories we consider will be at least abelian and therefore satisfy this assumption.

4.10 Definition/Proposition Let $\mathcal{C}$ be a $B T C$ and $(A, m, \eta)$ a commutative algebra, and let $(X, \mu),\left(X^{\prime}, \mu^{\prime}\right) \in$ ${ }_{A} \mathcal{C}$. Let $\alpha: X \otimes X^{\prime} \rightarrow X^{\prime \prime}$ be a coequalizer of the pair of morphisms

$$
\mu_{1}=\mu \otimes \operatorname{id}_{X^{\prime}}, \quad \mu_{2}=\left(\operatorname{id}_{X} \otimes \mu^{\prime}\right) \circ\left(c_{A, X} \otimes \operatorname{id}_{X^{\prime}}\right): A \otimes X \otimes X^{\prime} \rightarrow X \otimes X^{\prime} .
$$

By the universal property of $\alpha$, there is a unique $\mu^{\prime \prime}: A \otimes X^{\prime \prime} \rightarrow X^{\prime \prime}$ such that $\mu^{\prime \prime} \circ\left(\mathrm{id}_{A} \otimes \alpha\right)=\alpha \circ \mu_{1}=\alpha \circ \mu_{2}$. Now $\left(X^{\prime \prime}, \mu^{\prime \prime}\right) \in{ }_{A} \mathcal{C}$. With $(X, \mu) \otimes\left(X^{\prime}, \mu^{\prime}\right):=\left(X^{\prime \prime}, \mu^{\prime \prime}\right),{ }_{A} \mathcal{C}$ is a tensor category. (Commutativity of $A$ is needed for the interchange law $(s \otimes t) \circ\left(s^{\prime} \otimes t^{\prime}\right)=\left(s \circ s^{\prime}\right) \otimes\left(t \circ t^{\prime}\right)$ in ${ }_{A} \mathcal{C}$, to wit functoriality of $\otimes$ on morphisms.)

4.11 Lemma If $\mathcal{C}$ is braided and $(A, m, \eta)$ a commutative algebra in $\mathcal{C}$ then ${ }_{A} \mathcal{C}$ is a tensor category (with the above tensor product). The free module functor $F_{A}: \mathcal{C} \rightarrow{ }_{A} \mathcal{C}$ is a tensor functor.

We would like to prove that ${ }_{A} \mathcal{C}$ is braided or symmetric. This requires additional assumptions. If the algebra $(A, m, \eta)$ is étale, one can prove the following:

(i) If $\mathcal{C}$ is symmetric then ${ }_{A} \mathcal{C}$ is symmetric and the functor $F_{A}$ is symmetric.

(ii) If $A \in Z_{2}(\mathcal{C})$ then ${ }_{A} \mathcal{C}$ is braided and the functor $F_{A}$ is braided.

(iii) If $A \in Z_{2}(\mathcal{C})$ does not hold, the tensor category ${ }_{A} \mathcal{C}$ does not admit a braiding for which $F_{A}: \mathcal{C} \rightarrow{ }_{A} \mathcal{C}$ is braided. The reason is that, every object of ${ }_{A} \mathcal{C}$ being a quotient of $F_{A}(X)$ for some $X$, the only possible candidate for a braiding on ${ }_{A} \mathcal{C}$ making $F_{A}$ braided is the push-forward ' $F_{A}(c)$ ' of the braiding $c$ of $\mathcal{C}$. However, when $A \notin Z_{2}(\mathcal{C})$, the would-be braiding $F_{A}(c)$ is natural only w.r.t. one of its arguments. Reformulating this positively, one obtains: 
(iv) $F_{A}$ can always be considered as a braided tensor functor $\mathcal{C} \rightarrow Z_{1}\left({ }_{A} \mathcal{C}\right)$. More precisely, the tensor functor $F_{A}: \mathcal{C} \rightarrow{ }_{A} \mathcal{C}$ admits a central structure $\widehat{F_{A}}: \mathcal{C} \rightarrow Z_{1}\left({ }_{A} \mathcal{C}\right)$ in the sense of Definition 4.7. Cf. [29, 23].

(v) An $A$-module $(X, \mu)$ is called dyslectic [78, cf. also [54, or local when $\mu \circ c_{X, A}=\mu \circ \widetilde{c}_{X, A}$. The full subcategory ${ }_{A} \mathcal{C}^{0} \subset{ }_{A} \mathcal{C}$ of dyslectic modules is monoidal and in fact inherits a braiding from $\mathcal{C}$. (Notice that ${ }_{A} \mathcal{C}^{0}={ }_{A} \mathcal{C}$ when $A \in Z_{2}(\mathcal{C})$, thus in particular when $\mathcal{C}$ is symmetric.) The BTC ${ }_{A} \mathcal{C}^{0}$ will play an important rôle in the sequel.

(vi) In order to define a monoidal structure on ${ }_{A} \mathcal{C}$, where $A$ is an algebra in $\mathcal{C}$, one actually does not need a braiding on all of $\mathcal{C}$. Reviewing how the tensor structure was defined above, one realizes that it suffices to be able to commute $A$ with all objects of $\mathcal{C}$. More precisely, one should have a commutative algebra $\left(\left(A, e_{A}\right), m, \eta\right)$ in $Z_{1}(\mathcal{C})$ ! In this situation, one has a natural monoidal structure on ${ }_{A} \mathcal{C}$, cf. [83. (When $\mathcal{C}$ is braided and $A \in \mathcal{C} \stackrel{F_{1}}{\hookrightarrow} Z_{1}(\mathcal{C})$, this monoidal structure coincides with the one above since then $F_{1}(A)=\left(A, c_{A, \bullet}\right)$.) Furthermore, for a commutative algebra $A \in Z_{1}(\mathcal{C})$, Schauenburg proved [83] the remarkable braided equivalence $Z_{1}\left({ }_{A} \mathcal{C}\right) \simeq{ }_{A} Z_{1}(\mathcal{C})^{0}$.

\subsection{Duality in braided categories. Braided fusion categories}

Let $\mathcal{C}$ be a tensor category equipped with a left duality $X \mapsto\left({ }^{\vee} X, e_{X}, d_{X}\right)$ and a braiding $c$. Defining

$$
e_{X}^{\prime}=e_{X} \circ c_{X, \vee}: X \otimes{ }^{\vee} X \rightarrow \mathbf{1}, \quad d_{X}^{\prime}=\left(c_{X, \vee}\right)^{-1} \circ d_{X}: \mathbf{1} \rightarrow{ }^{\vee} X \otimes X,
$$

a computation shows that $\left({ }^{\vee} X, e_{X}^{\prime}, d_{X}^{\prime}\right)$ is a right dual for $X$. Thus in a braided category, left and right duals of each object are isomorphic, and

$$
\alpha_{X}=\left(\operatorname{id} \vee_{X} \otimes e_{X}\right) \circ\left(d_{X}^{\prime} \otimes \operatorname{id}_{X}\right): X \stackrel{\cong}{\rightarrow} \vee \vee X
$$

defines a natural isomorphism id $\cong \vee \vee-$ of functors. If $\mathcal{C}$ is symmetric a computation shows that $\alpha$ is a monoidal natural isomorphism, thus $\mathcal{C}$ is pivotal. Another computation shows that left and right traces coincide, thus $\mathcal{C}$ is spherical.

All this breaks down if $\mathcal{C}$ is braided but not symmetric, thus simply defining a right duality in terms of a given left duality and the braiding does not give a satisfactory result. One solution is to require in addition the existence of a ribbon structure:

4.12 Definition Let $\mathcal{C}$ be a (strict) tensor category with braiding $c$ and left duality $X \mapsto\left({ }^{\vee} X, e_{X}, d_{X}\right)$. A ribbon structure on $\mathcal{C}$ is a natural isomorphism $\Theta: \operatorname{id}_{\mathcal{C}} \rightarrow \operatorname{id}_{\mathcal{C}}$, i.e. a natural family of isomorphisms $\Theta_{X}: X \rightarrow X$, satisfying

$$
\begin{aligned}
\Theta_{X \otimes Y} & =\left(\Theta_{X} \otimes \Theta_{Y}\right) \circ c_{Y, X} \circ c_{X, Y} \quad \forall X, Y \\
\Theta_{\vee} & ={ }^{\vee}\left(\Theta_{X}\right) \quad \forall X .
\end{aligned}
$$

(Notice that when the braiding $c$ is symmetric, 4.4 makes the natural isomorphism $\Theta$ monoidal.) Using the ribbon structure, we modify the formulas 4.2 as follows:

$$
e_{X}^{\prime}=e_{X} \circ c_{X, \vee} \circ\left(\Theta_{X} \otimes \operatorname{id}{ }_{X}\right): X \otimes{ }^{\vee} X \rightarrow \mathbf{1}, \quad d_{X}^{\prime}=\left(\operatorname{id} \vee_{X} \otimes \Theta_{X}\right) \circ\left(c_{X, \vee}\right)^{-1} \circ d_{X}: \mathbf{1} \rightarrow{ }^{\vee} X \otimes X .
$$

Now one finds that $\left\{\alpha_{X}\right\}$, defined as in 4.3 , but using the modified definitions of $d_{X}^{\prime}, e_{X}^{\prime}$, is a monoidal natural isomorphism. Thus $\mathcal{C}$ is pivotal, and again in fact spherical. Cf. [49].

4.13 Remark Occasionally, it is preferable to reverse the above logic. Namely, if $\mathcal{C}$ is a spherical category and $c$ a braiding (with no compatibility assumed) then defining

$$
\Theta_{X}=\left(\operatorname{tr}_{X} \otimes \operatorname{id}_{X}\right)\left(c_{X, X}\right)
$$

one finds that $\left\{\Theta_{X}\right\}$ satisfies 4.44 .5 , thus is a ribbon structure compatible with the braiding $c$. Furthermore, the natural isomorphism $\alpha$ : id $\rightarrow \nabla$ - given as part of the spherical structure coincides with the one defined in terms of the left duality and $\Theta$ as in $(4.3)$. Therefore, for a braided category $(\mathcal{C}, c)$ with left duality, giving a pivotal (in fact spherical) structure $\alpha$ is equivalent to giving a ribbon structure $\Theta$ compatible with $c$. Cf. 98. 
We now briefly return to the subject of algebras in braided categories and their module categories. By a braided fusion category we simply mean a fusion category equipped with a braiding, and similarly for braided spherical categories. Now one has:

4.14 Proposition [54] If $\mathcal{C}$ is a braided fusion (resp. spherical) category and $A \in \mathcal{C}$ an étale algebra then ${ }_{A} \mathcal{C}$ is a fusion (resp. spherical) category (not necessarily braided) and

$$
\mathrm{FP} \operatorname{dim}_{A} \mathcal{C}=\frac{\mathrm{FP} \operatorname{dim} \mathcal{C}}{d(A)} .
$$

If $\mathcal{C}$ is spherical, both instances of FPdim in this identity can be replaced by dim as defined in terms of the spherical structures.

The dimension of the braided fusion category ${ }_{A} \mathcal{C}^{0}$ does not just depend on $(\mathrm{FP}) \operatorname{dim} \mathcal{C}$ and $d(A)$, but on 'how much' of the object $A$ lies in $Z_{2}(\mathcal{C})$. (E.g., it is evident that ${ }_{A} \mathcal{C}^{0}={ }_{A} \mathcal{C}$ when $A \in Z_{2}(\mathcal{C})$, giving the same dimension for both categories. But as we will see, it is also possible that ${ }_{A} \mathcal{C}^{0}$ is trivial, i.e. has dimension 1.) However, when $Z_{2}(\mathcal{C})$ is trivial, i.e. $\mathcal{C}$ is non-degenerate (resp. modular), one again has a simple formula, cf. (5.3) below.

As discussed earlier, the tensor functor $F_{A}: \mathcal{C} \rightarrow{ }_{A} \mathcal{C}$, while not braided in general, always admits a central structure. In the setting of fusion categories, central functors and module categories are closely related:

4.15 Theorem [23, Lemmas 3.5, 3.9] (i) If $\mathcal{C}$ is a braided fusion category, $\mathcal{D}$ is a fusion category and $F: \mathcal{C} \rightarrow \mathcal{D}$ a central functor then there exists a connected étale algebra $A \in \mathcal{C}$ such that the category ${ }_{A} \mathcal{C}$ is monoidally equivalent to the image of $F$, i.e. the smallest fusion subcategory of $\mathcal{D}$ containing $F(\mathcal{C})$. (The object $A$ is determined by $\operatorname{Hom}_{\mathcal{C}}(X, A) \cong \operatorname{Hom}_{\mathcal{D}}(F(X), \mathbf{1})$ and exists since $F$ has a right adjoint.)

(ii) If $\mathcal{C}$ is a braided fusion category and $A \in \mathcal{C}$ a connected étale algebra then the connected étale algebra $A^{\prime} \in \mathcal{C}$ obtained by (i) from the central functor $F_{A}: \mathcal{C} \rightarrow{ }_{A} \mathcal{C}$ is isomorphic to $A$.

\section{$5 \quad$ Modular categories}

\section{$5.1 \quad$ Basics}

The rest of this paper will be concerned with braided fusion categories over an algebraically closed field $k$, most often $\mathbb{C}$. Recall that every fusion category has a minimal fusion subcategory consisting only of the multiples of the unit 1. This subcategory is equivalent to Vect $_{k}$. A fusion category $\mathcal{C}$ is called trivial when it is itself equivalent to Vect ${ }_{k}$, which is the same as saying that every simple $X \in \mathcal{C}$ is isomorphic to $\mathbf{1}$.

\subsection{Definition $A$ braided fusion category $\mathcal{C}$ is called}

- pre-modular if it is spherical,

- non-degenerate if $Z_{2}(\mathcal{C})$ is trivial,

- modular if it is pre-modular and non-degenerate. Such a $\mathcal{C}$ is called just 'modular category' or MTC.

5.2 Remark 1. Non-degenerate braided fusion categories are related to symmetric fusion categories like von Neumann factors, i.e. von Neumann algebras $M$ with trivial center $Z(M)$, to commutative von Neumann algebras, where $Z(M)=M$. Since these two extremal types of von Neumann algebras play distinguished rôles in the general theory, it should not come as a surprise that the analogue also holds in the setting of braided fusion categories.

2. By an important theorem of Doplicher and Roberts [26] and independently Deligne [25], cf. Section 6 symmetric fusion categories are closely related to finite groups (and supergroups). Thus classifying symmetric fusion categories is essentially equivalent to classifying finite groups, a rather difficult task that has been achieved only partially. Given the importance of modular categories in the contexts of quantum group theory, conformal field theory, low dimensional topology, in particular topological quantum field theories, one may argue that the study and classification of modular categories is as natural and urgent as that of finite groups. 
Let $\mathcal{C}$ be a pre-modular category. For $X, Y \in \mathcal{C}$, define

$$
S(X, Y)=\operatorname{tr}_{X \otimes Y}\left(c_{Y, X} \circ c_{X, Y}\right) \in k .
$$

By the properties of the trace, $S(X, Y)$ depends only on the isomorphism classes $[X],[Y]$. Thus if $I(\mathcal{C})$ is the set of isomorphism classes of simple objects of $\mathcal{C}$ and we choose representers $X_{i}$, we can define an $|I(\mathcal{C})| \times|I(\mathcal{C})|$ matrix $S$ by $S_{i, j}=S\left(X_{i}, X_{j}\right) . S$ is symmetric, and it is easy to see that non-triviality of $Z_{2}(\mathcal{C})$ implies singularity of $S$ : If $X_{i} \in Z_{2}(\mathcal{C})$ then $S_{i, j}=\operatorname{tr}_{X_{i} \otimes X_{j}}(\mathrm{id})=d\left(X_{i}\right) d\left(X_{j}\right)$ for all $j$, and thus the $i$-th row (and column) are proportional to the 0 -th row (column) (where $X_{0} \cong 1$ ).

More interestingly, for $\mathcal{C}$ pre-modular one can show, cf. 86, 87, 80:

- For simple $X, Y$, one has $S(X, Y)=d(X) d(Y)$ if and only if $X$ and $Y$ commute.

- Let $\mathcal{K} \subset \mathcal{C}$ be a fusion subcategory. Then

$$
\sum_{i \in I(\mathcal{K})} d\left(X_{i}\right) S_{i, j}=\left\{\begin{array}{cc}
d\left(X_{j}\right) \operatorname{dim} \mathcal{K} & \text { if } X_{j} \in \mathcal{K}^{\prime} \\
0 & \text { otherwise }
\end{array}\right.
$$

- If $Z_{2}(\mathcal{C})$ is trivial then $S^{2}=\operatorname{dim} \mathcal{C} C$, where $C$ is the 'charge-conjugation' matrix: $C_{i, j}=\delta_{i, \bar{j}}$. (Note that $C^{2}=1$.) Thus if we assume $\operatorname{dim} \mathcal{C} \neq 0$ (which is automatic over $\mathbb{C}[32]$ ), then $S$ is invertible if and only if $Z_{2}(\mathcal{C})$ is trivial, i.e. $\mathcal{C}$ is modular.

- If $\mathcal{C}$ is modular then the 'Gauss sums' of $\mathcal{C}$, defined by

$$
\Omega^{ \pm}(\mathcal{C})=\sum_{i \in I(\mathcal{C})} \Theta\left(X_{i}\right)^{ \pm 1} d\left(X_{i}\right)^{2}
$$

satisfy $\Omega^{+}(\mathcal{C}) \Omega^{-}(\mathcal{C})=\operatorname{dim} \mathcal{C}$ and the diagonal $|I(\mathcal{C})| \times|I(\mathcal{C})|$-matrix $T$ defined by $T_{i, j}=\delta_{i, j} \Theta\left(X_{i}\right)$ satisfies $T S T S T=\Omega^{+}(\mathcal{C}) S$. (The modular category $\mathcal{C}$ is called 'anomaly-free' when $\Omega^{+}=\Omega^{-}$.)

- Therefore, if $\mathcal{C}$ is modular, thus $S$ invertible, we have $S^{2}=\alpha C$ and $(S T)^{3}=\beta C$ with $\alpha \beta \neq 0$. This means that $S$ and $T$ define a projective representation of the modular group $S L(2, \mathbb{Z})$. (Recall that the latter is generated by the elements

$$
s=\left(\begin{array}{cc}
0 & 1 \\
-1 & 0
\end{array}\right), \quad t=\left(\begin{array}{ll}
1 & 1 \\
0 & 1
\end{array}\right),
$$

which satisfy $s^{2}=-\mathbf{1},(s t)^{3}=\mathbf{1}$.) The existence of this representation is the rationale behind the terminology 'modular categories'.

Let $X$ be a simple object and $Y$ invertible. Then $X \otimes Y$ is simple, thus $c_{Y, X} \circ c_{X, Y} \in \operatorname{Aut}(X \otimes Y)$ is a scalar (=element of the ground field). Multiplying this scalar by $d(X)$ gives $S(X, Y)$, but this is not the point. The point is that the map $f: I \times I_{1} \rightarrow k$, where $I_{1} \subset I$ is the subgroup of invertible isomorphism classes, obtained by the above consideration is a homomorphism w.r.t. the second argument. One can show that $f(Z, L)=f(X, L) f(Y, L)$ whenever $N_{X, Y}^{Z}>0$, cf. [80, Section 4]. This implies $f(X, Z)=f(Y, Z)$ whenever $\partial X=\partial Y$, where $\partial: I \rightarrow G(I)$ is the universal group grading discussed in Section 3.2 Thus $f$ descends to a bihomomorphism $G(I) \times I_{1} \rightarrow k$. Remarkably one has:

5.3 Theorem [42] When $\mathcal{C}$ is modular, the above map is a non-degenerate pairing, establishing a canonical isomorphism $I_{1} \rightarrow \widehat{G(I)}$.

5.4 REMARK Recall that for a finite group $H$, the grading group of $\operatorname{Rep} H$ is given by $G(\widehat{H}) \cong \widehat{Z(H)}$. On the other hand, $I_{1}(\operatorname{Rep} H) \cong \widehat{H_{a b}}$, where $H_{a b}=H /[H, H]$ is the abelianization. The abelian groups $Z(H)$ and $H_{a b}$ have little to do with each other. In view of this, Theorem 5.3 is one of many manifestations of the observation of [80] that a modular category is "a self-dual object that is more symmetric than a group".

In the next two sections, we will encounter several constructions that give rise to modular categories. However, it seems instructive to give an example already at this point.

5.5 ExAmple Let $A$ be a finite abelian group and $\widehat{A}$ its character group. Let $\mathcal{C}_{0}(A)$ be the strict tensor category defined by 
(i) $\operatorname{Obj} \mathcal{C}(A)=A \times \widehat{A}$.

(ii) $\operatorname{Hom}\left((g, \phi),\left(g^{\prime}, \phi^{\prime}\right)\right)=\mathbb{C}$ if $(g, \phi)=\left(g^{\prime}, \phi^{\prime}\right)$ and $=\{0\}$ otherwise.

(iii) $(g, \phi) \otimes\left(g^{\prime}, \phi^{\prime}\right)=\left(g g^{\prime}, \phi \phi^{\prime}\right)$. Composition and tensor product of morphisms are defined as multiplication of complex numbers.

(iv) The braiding is given by $c_{(g, \phi),\left(g^{\prime}, \phi^{\prime}\right)}=\phi\left(g^{\prime}\right)$ id. (This makes sense since $(g, \phi) \otimes\left(g^{\prime}, \phi^{\prime}\right)=\left(g^{\prime}, \phi^{\prime}\right) \otimes(g, \phi)$.)

Now $\mathcal{C}(A)$ is the closure of $\mathcal{C}_{0}(A)$ w.r.t. direct sums. One finds $\Theta((g, \phi))=\phi(g)$ and $S\left((g, \phi),\left(g^{\prime}, \phi^{\prime}\right)\right)=$ $\phi\left(g^{\prime}\right) \phi^{\prime}(g)$, from which it follows easily that $\left(e, \phi_{0}\right)$, where $\phi_{0} \equiv 1$, is the only central object, thus $\mathcal{C}(A)$ is modular.

The above construction of $\mathcal{C}(A)$ admits generalization to non-abelian finite groups, but that is better done using Hopf algebra language. This leads to the quantum double $D(G)$ of a finite group or a finite dimensional Hopf algebra $D(H)$, cf. the first paragraph of Section 7 .

5.6 For any finite abelian group, one easily proves that $\Omega^{ \pm}(\mathcal{C}(A))=N$. Thus the categories $\mathcal{C}(A)$ are anomalyfree. If $N$ is odd then the full subcategory $\mathcal{D}_{N} \subset \mathcal{C}(\mathbb{Z} / N \mathbb{Z})$ with objects $\{(k, k) \mid k=0, \ldots, N-1\}$ is itself modular, cf. Remark 5.22 , 3 , and one has

$$
\Omega^{ \pm}\left(\mathcal{D}_{N}\right)=\sum_{k=0}^{N-1} e^{ \pm \frac{2 \pi i}{N} k^{2}},
$$

which is a classical Gauss sum. (This motivates calling the quantities $\Omega^{ \pm}(\mathcal{C})$ 'Gauss sums' in general.) By the classical computation of these Gauss sums, we find that $\Omega^{+}\left(\mathcal{D}_{N}\right)$ equals $\sqrt{N}$ when $N \equiv 1(\bmod 4)$ and $i \sqrt{N}$ when $N \equiv 3(\bmod 4)$. In view of $\Omega^{-}(\mathcal{C})=\overline{\Omega^{+}(\mathcal{C})}$, the categories $\mathcal{D}_{N}$ with $N \equiv 3(\bmod 4)$ therefore provide our first examples of modular categories that are not anomaly-free.

5.7 Modular categories first appeared explicitly in [86], but some aspects of that paper were anticipated by two years in [80, which also was inspired by [62] but in addition drew upon the well established operator algebraic approach [44 to axiomatic quantum field theory and in particular on 35. In particular, 80, contains the first proof of the equivalence between invertibility of the $S$-matrix, in terms of which modularity was defined in [86, 87, and triviality of $Z_{2}(\mathcal{C})$. (For a more general recent proof cf. [13.)

\subsection{Digression: Modular categories in topology and mathematical physics}

5.8 While some inspiration for [86] came from conformal field theory and in particular [62], cf. below, the main motivation came from low-dimensional topology. In 1988/9, Witten [95] had proposed an interpretation of the new 'quantum invariants' of knots and 3-manifolds (in particular Jones' polynomial knot invariant) in terms of 'topological quantum field theories' (TQFTs), defined via a non-rigorous (not-yet-rigorous?) pathintegral formalism. From this work, Atiyah 2 immediately abstracted the mathematical axioms that should be satisfied by a TQFT, and Reshetikhin and Turaev [81] soon used the representation theory of quantum groups to rigorously construct a TQFT that should essentially be that studied by Witten. The aim of [86] was then to isolate the mathematical structure that is behind the construction in 81] as to enable generalizations, and indeed one has a $2+1$-dimensional TQFT $F_{\mathcal{C}}$ for each modular category $\mathcal{C}$, wherever $\mathcal{C}$ may come from. (Cf. 87] for a full exposition of the early work on the subject and [5] for a somewhat more recent introduction.)

Since there is no space for going into this subject to any depth, we limit ourselves to mentioning that a TQFT in $2+1$ dimensions gives rise to projective representations of the mapping class groups of all closed two-manifolds. The mapping class group of the 2 -torus is the modular group $S L(2, \mathbb{Z})$, and its projective representation produced by the TQFT $F_{\mathcal{C}}$ associated with the modular category $\mathcal{C}$ is just the one encountered above in terms of $S$ and $T$.

5.9 Before we turn to a brief discussion of the rôle of modular categories in conformal field theory, we mention another manifestation of them in mathematical physics. It has turned out that infinite quantum systems (both field theories and spin systems) in two spatial dimensions can have 'topological excitations', whose mathematical analysis leads to braided tensor categories which often turn out to be modular. An important example is Kitaev's 'toric code' [55], a quantum spin system in two dimensions which gives rise to the (rather simple) modular category $D(\mathbb{Z} / 2 \mathbb{Z})-\operatorname{Mod}($ the $\mathcal{C}(\mathbb{Z} / 2 \mathbb{Z})$ of Example 5.5). (Cf. also [73.) There is a generalization of the toric code to finite groups other than $\mathbb{Z} / 2 \mathbb{Z}$, but not everything has been worked out yet. The toric code models play a prominent rôle in the subject of topological quantum computing, reviewed e.g. in [36, 75, 93. 
5.10 While Kitaev's model lives in $2+1$ dimensions and has a mass gap, braided and modular categories also arise in conformally invariant (thus massless) quantum field theories in $1+1$ or $2+0$ dimensions, a subject that has been researched very extensively. In particular, there have been two rigorous and model-independent proofs of the statement that suitable chiral conformal field theories have modular representation theories. In the operator algebraic approach to CFTs, the basic definitions are quite easy to state, and we briefly do so.

The group $\mathcal{P}=P S U(1,1)$ acts on $S^{1}=\{z \in \mathbb{Z}|| z \mid=1\}$ by $\left(\begin{array}{ll}a & b \\ c & d\end{array}\right) z=\frac{a z+b}{c z+d}$. Let $\mathcal{I}$ be the set of connected open subsets $I \subset S^{1}$ such that $\emptyset \neq I \neq S^{1}$. (Thus $\mathcal{I}$ consists of the non-trivial connected open intervals in $S^{1}$.) If $I \in \mathcal{I}$ and $g \in \mathcal{P}$ then $g I \in \mathcal{I}$.

5.11 Definition $A$ chiral $C F T \mathcal{A}$ is a quadruple $\left(H_{0}, A(\cdot), U, \Omega\right)$, where $H_{0}$ is a separable Hilbert space (thus essentially independent of $\mathcal{A}), \Omega \in H$ a unit vector, $U$ is a strongly continuous positive-energy representation of the group $P S U(1,1)$ on $H$, and for each $I \in \mathcal{I}, A(I) \subset B\left(H_{0}\right)$ is a von Neumann algebra. These data must satisfy the following axioms:

(i) 'Isotony': $I_{1} \subset I_{2} \Rightarrow A\left(I_{1}\right) \subset A\left(I_{2}\right)$

(i) 'Locality': $I_{1} \cap I_{2}=\emptyset \Rightarrow\left[A\left(I_{1}\right), A\left(I_{2}\right)\right]=\{0\}$.

(iii) 'Irreducibility': $\cap_{I} A(I)^{\prime}=\mathbb{C} 1$.

(iv) 'Möbius Covariance': If $I \in \mathcal{I}, g \in \mathcal{P}$ then $U(g) A(I) U(g)^{*}=A(g I)$.

(v) $U(g) \Omega=\Omega \forall g \in \mathcal{P}$.

Already from these few axioms one can prove a number of important results, among which:

(a) 'Factoriality': Each $A(I)$ is a factor, i.e. has trivial center.

(b) 'Weak additivity': If $I_{1}, I_{2} \in \mathcal{I}$ such that $I_{1} \cup I_{2} \in \mathcal{I}$ then $A\left(I_{1}\right) \vee A\left(I_{2}\right)=A\left(I_{1} \cup I_{2}\right)$.

(c) 'Haag duality': For each $I \in \mathcal{I}$ one has $A(I)^{\prime}=A\left(I^{\prime}\right)$, where $I^{\prime}$ is the interior of $S^{1}-I$.

One has a notion of representation for chiral CFTs:

5.12 Definition Let $\mathcal{A}=(H, A(\cdot), U, \Omega)$ be a chiral CFT. A representation of $\mathcal{A}$ is a pair $\left(H,\left\{\pi_{I}\right\}_{I \in \mathcal{I}}\right)$, where $H$ is a (separable) Hilbert space and $\left\{\pi_{I}: A(I) \rightarrow B(H)\right\}_{I \in \mathcal{I}}$ is a family of $*$-representations satisfying $\pi_{I_{2}}\left\lceil A\left(I_{1}\right)=\pi_{I_{1}}\right.$ whenever $I_{1} \subset I_{2}$. A morphism of representations $\left(H,\left\{\pi_{I}\right\}\right),\left(H^{\prime},\left\{\pi_{I}^{\prime}\right\}\right)$ is a bounded operator $V: H \rightarrow H^{\prime}$ such that $V \pi_{I}(\cdot)=\pi_{I}^{\prime}(\cdot) V$ for each $I \in \mathcal{I}$. This defines a $*$-category $\operatorname{Rep} \mathcal{A}$.

If $\left(H,\left\{\pi_{I}\right\}\right)$ is a representation, we define $\operatorname{dim} \pi=\left[\pi_{I^{\prime}}\left(A\left(I^{\prime}\right)^{\prime}\right): \pi_{I}(A(I))\right] \in[1, \infty]$, where the brackets denote the Jones index, and the r.h.s. is independent of $I \in \mathcal{I}$. $\operatorname{Rep}_{f} \mathcal{A}$ denotes the full subcategory of $\operatorname{Rep} \mathcal{A}$ of representations with $\operatorname{dim} \pi<\infty$.

It is clear that $\operatorname{Rep} \mathcal{A}$ and $\operatorname{Rep}_{f} \mathcal{A}$ are $*$-categories. But one prove much more: Both admit canonical braided monoidal structures, and $\operatorname{Rep}_{f} \mathcal{A}$ is semisimple with (two-sided) duals, but not necessarily finite. Cf. [35, II]. In order to prove finiteness or modularity of $\operatorname{Rep}_{F} \mathcal{A}$ one needs to assume more:

5.13 Definition A chiral $C F T \mathcal{A}=(H, A(\cdot), U, \Omega)$ is called completely rational if the following holds:

(i) 'Split property': If $I_{1}, I_{2} \in \mathcal{I}$ satisfy $\overline{I_{1}} \cap \overline{I_{2}}=\emptyset$ then the natural map $A\left(I_{1}\right) \otimes_{\text {alg }} A\left(I_{2}\right) \rightarrow A\left(I_{1}\right) \vee A\left(I_{1}\right)$ induces an isomorphism of von Neumann algebras.

(ii) 'Strong additivity': If $I_{1}, I_{2} \in \mathcal{I}$ satisfy $\#\left(\overline{I_{1}} \cap \overline{I_{2}}\right)=1$ (i.e. the intervals are disjoint but adjacent) then $A\left(I_{1}\right) \vee A\left(I_{2}\right)=A(I)$, where $I$ is the interior of the closure of $I_{1} \cup I_{2}$.

(iii) 'Finiteness': Let $I_{1}, I_{2} \in \mathcal{I}$ such that $\overline{I_{1}} \cap \overline{I_{2}}=\emptyset$. Then $\left(I_{1} \cup I_{2}\right)^{\prime}=I_{3} \cup I_{4}$ for certain $I_{3}, I_{4} \in \mathcal{I}$. The quantity $\mu(A)=\left[\left(A\left(I_{3}\right) \vee A\left(I_{4}\right)^{\prime}\right)^{\prime}: A\left(I_{1}\right) \vee A\left(I_{2}\right)\right]$ (which a priori is in $[1, \infty]$ and can be shown to be independent of $\left.I_{1}, I_{2}\right)$ is finite.

(Axiom (ii) is not really essential.) The following was proven in [51]:

5.14 Theorem If $\mathcal{A}=(H, A(\cdot), U, \Omega)$ is a completely rational chiral $C F T$ then $\operatorname{Rep}_{f} \mathcal{A}$ is modular with $\operatorname{dim} \operatorname{Rep}_{f} \mathcal{A}=\mu(A)$. 
Many examples of completely rational chiral CFTs are known, and Theorem 5.14 is just the beginning of a rapidly growing theory. (The fact that the dimension of the representation category can (in principle) be obtained by looking at just a few local algebras is extremely useful.) Cf. e.g. the references in [71].

Around 2005, Huang proved a similar result in the framework of vertex operator algebras, cf. [45] and references therein, assuming the property of ' $C_{2}$-cofiniteness', which is similar to (iii) in Definition 5.13 .

The most important chiral conformal field theories are related to (a) the projective 'positive energy' representations of the diffeomorphism group $\operatorname{Diff}^{+}\left(S^{1}\right)$ of the circle and/or its (centrally extended) Lie algebra, the Virasoro algebra, and (b) the positive energy representations of the loop groups $C^{\infty}\left(S^{1}, G\right)$, where $G$ is a compact Lie group. These representation categories can be studied without reference to conformal field theory, cf. e.g. 79, 92, but defining the tensor structure does require conformal field theory (or at least techniques of the latter), like those of [35] or 94].

5.15 For any algebraically closed field $k$, the group $H^{2}\left(S L(2, \mathbb{Z}), k^{*}\right)$ is trivial. This implies that by rescaling the matrices $S, T$ one can obtain a true representation of the modular group, and there are exactly six ways of doing this. There are two situations where there is a preferred choice: When $\mathcal{C}$ is anomaly-free, as when $\mathcal{C} \simeq Z_{1}(\mathcal{D})$, one has $\Omega^{+}=\Omega^{-}= \pm \operatorname{dim} \mathcal{C}$. Then the renormalization $S^{\prime}=S / \Omega^{+}, T^{\prime}=T$ gives a true representation of $S L(2, \mathbb{Z})$, which may be considered more canonical than the others.

On the other hand, when the modular category arises as the representation category of a conformal field theory $\mathcal{A}$ (in the setting of operator algebras or vertex algebras), for each (equivalence classes) of simple objects of $\operatorname{Rep} \mathcal{A}$, there is an analytic function $f_{i}: \mathbb{H} \rightarrow \mathbb{C}$, the 'character' of that representation. Collecting these $n=|I(\operatorname{Rep} \mathcal{A})|$ functions in a vector-valued function $f: \mathbb{H} \rightarrow V=\mathbb{C}^{n}$, one finds that $f$ satisfies the following definition:

5.16 Definition Let $V$ be a finite dimensional complex vector space and $\pi: S L(2, \mathbb{Z}) \rightarrow \operatorname{End}(V)$ a representation. A vector valued modular form of type $\pi$ is a holomorphic map $\rho: \mathbb{H} \rightarrow V$ satisfying

$$
f\left(g^{-1} z\right)=\pi(g) f(z) \quad \forall z \in \mathbb{H}, g \in S L(2, \mathbb{Z}) .
$$

Here $\mathbb{H}=\{z \in \mathbb{C} \mid \operatorname{Im} z>0\}$ and $S L(2, \mathbb{Z})$ acts on $\mathbb{H}$ by $\left(\begin{array}{ll}a & b \\ c & d\end{array}\right) z=\frac{a z+b}{c z+d}$.

Here, $\pi$ is a uniquely determined (by the CQFT) true representation of $S L(2, \mathbb{C}$ ) obtained by a particular renormalization the matrices $S, T$ associated with the modular category $\operatorname{Rep} \mathcal{A}$.

In the CQFTs associated with the representation theories of the Virasoro and the Kac-Moody algebras, the above vector valued modular forms can be studied very explicitly, cf. e.g. [92].

5.17 In all rational chiral CFTs that have been studied explicitly, it turned out that there is an $N \in \mathbb{N}$ such that all conformal characters $\chi_{i}$ are modular functions for the congruence subgroup $\Gamma(N)=\{M \in S L(2, \mathbb{Z}) \mid M \equiv \mathbf{1}$ ( $\bmod N)\}$. This means that $\chi_{i}(g z)=\chi_{i}(z)$ for all $g \in \Gamma(N)$ and all $i$. In view of $(5.2)$ this amounts to $\Gamma(N) \subset \operatorname{ker}(\pi)$. This led to the folk conjecture that this 'congruence subgroup property' holds in all rational chiral CFTs. Such a general result was indeed obtained in 8] (where unfortunately no rigorous formalism of CFTs was used). The 'conductor' $N$ is closely related to the order of the diagonal matrix $T$ (all elements of which are roots of unity). It is natural to ask whether a similar result can be proven for all modular categories irrespective of whether they arise from a chiral CFT. (The latter question is an important open problem.) The answer is yes, but before we state it, we revert back to 'pure' mathematics.

\subsection{Modular categories: Structure theory and module categories}

The work 8 inspired 84 , where a congruence subgroup theorem was proven for the modular categories of the form $D(H)$ - Mod and finally the very recent [76] with a result valid for all modular categories:

5.18 Theorem [76] (a) If $\mathcal{D}$ is a spherical fusion category and $\mathcal{C}=Z_{1}(\mathcal{D})$ then the kernel of the canonical (cf. $5.15)$ true representation of $S L(2, \mathbb{Z})$ contains $\Gamma(N)$, where $N$ is the 'Frobenius-Schur exponent' of $\mathcal{C}$.

(b) If $\mathcal{C}$ is an arbitrary modular category, then $\Gamma(N)$ is contained in the kernel of the canonical projective representation of $S L(2, \mathbb{Z})$ generated by $S, T$.

The following results from [67, generalized to non-degenerate braided fusion categories in [30, are the first steps towards a structure theory and perhaps classification of modular categories. Part (iv) shows that, in a sense, modular categories are better behaved than finite groups! 
5.19 Theorem Let $\mathcal{C}$ be a modular category and $\mathcal{D}_{1} \subset \mathcal{C}$ a fusion subcategory. Let $\mathcal{D}_{2}=C_{\mathcal{C}}\left(\mathcal{D}_{1}\right)$. Then

(i) $\operatorname{dim} \mathcal{D}_{1} \cdot \operatorname{dim} \mathcal{D}_{2}=\operatorname{dim} \mathcal{C}$.

(ii) $C_{\mathcal{C}}\left(\mathcal{D}_{2}\right)=\overline{\mathcal{D}_{1}}$, where the r.h.s. denotes the closure of $\mathcal{D}_{1}$ under isomorphisms (i.e. the smallest replete fusion subcategory of $\mathcal{C}$ containing $\left.\mathcal{D}_{1}\right)$. Thus if $\mathcal{D} \subset \mathcal{C}$ is a replete fusion subcategory then $\mathcal{D}^{\prime \prime}=\mathcal{D}$.

(iii) If $\mathcal{D} \subset \mathcal{C}$ is a full tensor subcategory then $Z_{2}(\mathcal{D})=Z_{2}\left(\mathcal{D}^{\prime}\right)$. In particular, if $\mathcal{D}$ is modular then so is $\mathcal{D}^{\prime}$.

(iv) If $\mathcal{D} \subset \mathcal{C}$ is modular then $\mathcal{C} \simeq \mathcal{D} \otimes \mathcal{D}^{\prime}$ as $B T C$.

5.20 Definition A modular category $\mathcal{C}$ is called prime if every modular fusion subcategory is either trivial or equivalent to $\mathcal{C}$.

5.21 Corollary Every modular category is equivalent to a finite direct product of prime modular categories.

5.22 Remark 1. Statement (ii) is similar to the 'double commutant theorem' in the theory of von Neumann algebras: The commutant $M^{\prime \prime}$ of the commutant of a von Neumann algebra equals $M$. Statement (iv), according to which modular subcategories always are direct factors, is analogous to the fact that an inclusion $N \subset M$ of type I factors gives rise to an isomorphism $M \cong N \otimes N^{\prime}$.

2. If $G$ is a finite non-abelian simple group then the modular category $D(G)$ - Mod is prime. In fact it contains only one fusion subcategory, namely $\operatorname{Rep} G$. It follows that the classification of prime modular categories contains the classification of finite simple (non-abelian) groups! For general finite groups, $D(G)$ - Mod can have many fusion subcategories. Cf. [74 for a classification.

3. In general, the prime factorization of a modular category is not unique. An example is already provided by $\mathcal{C}=D(A)-\operatorname{Mod}$, where $A=\mathbb{Z} / p \mathbb{Z}$ is cyclic of prime order $p \neq 2$. In this case, the replete prime modular subcategories of $\mathcal{C}$ are labeled by the isomorphisms $\phi: A \rightarrow \widehat{A}$, and $\left(\mathcal{D}_{\phi}\right)^{\prime}=\mathcal{D}_{\bar{\phi}}$ where $\bar{\phi}(\cdot)=\phi(\cdot)^{-1}$. Cf. 67 . In this example, all objects of $\mathcal{C}$ are invertible, which is crucial for the non-uniqueness: By [23, Proposition 2.2], the prime factorization of $\mathcal{C}$ is unique if $\mathcal{C}$ has no invertible objects apart from 1 . More generally, prime factors having no invertibles other than $\mathbf{1}$ appear identically in every prime factorization of $\mathcal{C}$. Thus the non-uniqueness results from the possibility of 'moving invertible objects from one direct factor to another'. The details have not yet been clarified, but it is clear that homomorphisms $\phi: G(\mathcal{C}) \rightarrow I_{1}(\mathcal{C}) \cong \widehat{G(\mathcal{C})}$ play a rôle. (Recall from 5.3 that the abelian grading group $G(\mathcal{C})$ and the group $I_{1}(\mathcal{C})$ of (isomorphism classes of) invertible objects of $\mathcal{C}$ are canonically dual to each other.)

If $\mathcal{C}$ is modular and $\mathcal{D} \subset \mathcal{C}$ a fusion subcategory then $Z_{2}(\mathcal{D})=\mathcal{D} \cap \mathcal{D}^{\prime} \subset \mathcal{C} \cap \mathcal{D}^{\prime}=C_{\mathcal{C}}(\mathcal{D})$, implying

$$
\operatorname{dim} \mathcal{C}=\operatorname{dim} \mathcal{D} \cdot \operatorname{dim} C_{\mathcal{C}}(\mathcal{D}) \geq \operatorname{dim} \mathcal{D} \cdot \operatorname{dim} Z_{2}(\mathcal{D}) .
$$

This motivated the conjecture [67]:

5.23 Conjecture If $\mathcal{D}$ is a pre-modular category, there is a modular category $\mathcal{C}$ containing $\mathcal{D}$ as a fusion subcategory such that $\operatorname{dim} \mathcal{C}=\operatorname{dim} \mathcal{D} \cdot Z_{2}(\mathcal{D})$.

(There are indications that the conjecture in this generality may be false, but see Theorem 6.5 below.)

Above, we pointed out the analogy between non-degenerate braided fusion categories and von Neumann factors. This analogy goes a bit further: Since factors are simple (as algebras), their homomorphisms have trivial kernels and therefore are embeddings. Analogously, one has:

5.24 Proposition [23, Corollary 3.26] Any braided tensor functor $F: \mathcal{C} \rightarrow \mathcal{D}$ between braided fusion categories with $\mathcal{C}$ (almost) non-degenerate is fully faithful.

Sketch of Proof. Replacing $\mathcal{D}$ by its subcategory $F(\mathcal{C})$, we may assume that $F$ is surjective. By results mentioned earlier, there is an étale algebra $A \in \mathcal{C}$ and an equivalence $H: \mathcal{D} \rightarrow{ }_{A} \mathcal{C}$ of fusion categories such that $H \circ F \cong F_{A}$. That $F_{A}$ is braided implies $A \in Z_{2}(\mathcal{C})$, and $\mathcal{C}$ being non-degenerate, we have $A=\mathbf{1}$, thus $F \cong$ id.

In analogy to Proposition 4.14 one has the following result concerning dyslectic module categories for étale algebras in non-degenerate braided fusion categories: 
5.25 TheOrem [54] Let $\mathcal{C}$ be a non-degenerate braided fusion category and $A \in \mathcal{C}$ a connected étale algebra. Then the dyslectic module category ${ }_{A} \mathcal{C}^{0}$ is a non-degenerate braided fusion category and

$$
\mathrm{FP} \operatorname{dim}{ }_{A} \mathcal{C}^{0}=\frac{\mathrm{FP} \operatorname{dim} \mathcal{C}}{d(A)^{2}} .
$$

If $\mathcal{C}$ is spherical, so is ${ }_{A} \mathcal{C}^{0}$ and FPdim can be replaced by dim.

In the next two sections we discuss two ways of constructing modular categories (or, more generally, nondegenerate braided fusion categories). The first, modularization, starts from a pre-modular category and the second from a mere (spherical) fusion category.

\section{Modularization of pre-modular categories. Generalizations}

Let $\mathcal{C}$ be a pre-modular category, to wit a braided spherical fusion category. Its symmetric center $Z_{2}(\mathcal{C})$ then is a symmetric spherical fusion category. Since non-modularity of $\mathcal{C}$ is equivalent to $Z_{2}(\mathcal{C})$ being non-trivial, it is natural to try to 'quotient out' the full subcategory $Z_{2}(\mathcal{C})$ in order to obtain a modular category ' $\mathcal{C} / Z_{2}(\mathcal{C})$ '. Formalizing this idea one arrives at the following:

6.1 Definition [18] A modularization of a pre-modular category $\mathcal{C}$ is a functor $F: \mathcal{C} \rightarrow \mathcal{D}$ of braided fusion categories, where $\mathcal{D}$ is modular and $F$ is surjective (or 'dominant') in the sense that every object of $\mathcal{D}$ is a subobject of one of the form $F(X)$ with $X \in \mathcal{C}$.

The fact that $F$ is supposed to be braided and surjective implies that it must trivialize $Z_{2}(\mathcal{C})$, i.e. $F(X)$ must be a multiple of the identity whenever $X \in Z_{2}(\mathcal{C})$. The following was shown in [18, 64]:

6.2 Theorem A pre-modular category admits a modularization if and only if the symmetric category $Z_{2}(\mathcal{C})$ is purely even, i.e. all objects have trivial twist, i.e. $\theta_{X}=\operatorname{id}_{X} \forall X$.

The proof relies on the following deep result:

6.3 Theorem [26, 25] Let $\mathcal{C}$ be a spherical symmetric fusion category with trivial twists. Then there is a finite group $G$, unique up to isomorphism, such that $\mathcal{C} \simeq \operatorname{Rep} G$ as symmetric tensor category. This equivalence is unique up to natural monoidal isomorphism.

(Both [26, 25] prove much more general results. In [26], $\mathcal{C}$ is required to be unitary.) Now, if $G$ is a finite group, the vector space $A=\operatorname{Fun}(G, \mathbb{C})$ underlying the left regular representation also is a commutative algebra, and one finds that $A$ is a connected étale algebra in $\operatorname{Rep} G$. Furthermore, the module category ${ }_{A}(\operatorname{Rep} G)$ is trivial. We call $A$ the regular algebra, and we also do this for the corresponding object in $\mathcal{C}$ when Theorem 6.3 is invoked. Combining this observation with Theorem 6.3 it is clear how to obtain a modularization of a pre-modular category $\mathcal{C}$ with trivial twists: Take $\mathcal{D}={ }_{A} \mathcal{C}$, where $A$ is the regular algebra of the symmetric even category $Z_{2}(\mathcal{C}) \subset \mathcal{C}$, and $F=F_{A}$. It is not hard to show that $\mathcal{D}$ indeed is modular.

If $Z_{2}(\mathcal{C})$ contains odd/Fermionic objects, i.e. objects with $\Theta_{X}=-\mathrm{id}_{X}$, the above approach does not work. For a symmetric spherical category $\mathcal{C}$ with non-trivial twists, one has a generalization of Theorem 6.3, giving rise to a finite group $G$ together with an element $k \in Z(G)$ of order two. (Such a pair $(G, k)$ is occasionally called a super-group.) One still has an equivalence $\mathcal{C} \simeq \operatorname{Rep} G$ of fusion categories, but the braidings of $\mathcal{C}$ and $\operatorname{Rep} G$ are related by the Koszul-type rule $c_{\mathcal{C}}(X, Y)= \pm c_{\operatorname{Rep} G}(X, Y)$ for simple $X, Y$, where the minus sign applies when $X$ and $Y$ are both odd, cf. [26, Section 7]. The regular representation of a super-group $(G, k)$ again is a connected separable algebra $A \in \operatorname{Rep}(G, k)$, but it is only graded commutative. As a consequence, when $\mathcal{C}$ is a braided fusion category containing $\operatorname{Rep}(G, k)$ as a full subcategory, the module category ${ }_{A} \mathcal{C}$ is not a $k$-linear tensor category but a tensor category enriched over the category SVect ${ }_{k}$ of super vector spaces. (Notice that, by contrast to tensor categories enriched over Vect, such a category is not a tensor category, since the interchange law holds only up to signs: $(s \otimes t) \circ\left(s^{\prime} \otimes t^{\prime}\right)= \pm\left(s \circ s^{\prime}\right) \otimes\left(t \circ t^{\prime}\right)$.)

However, if $(G, k)$ is a super-group, $\{e, k\}$ is a normal subgroup and if $H$ denotes the quotient group, $\operatorname{Rep}(G, k)$ contains the even category $\operatorname{Rep} H$ as a full fusion subcategory. If $A \in \operatorname{Rep} H$ is the regular étale algebra of $H$, one finds ${ }_{A}(\operatorname{Rep}(G, k)) \cong$ SVect, the category of super-vector spaces, to wit the representation category of the super-group $(\{e, k\}, k)$. This shows that every pre-modular category $\mathcal{C}$ admits a surjective braided functor $F: \mathcal{C} \rightarrow \mathcal{D}$, where $\mathcal{D}$ is 'almost-modular': 
6.4 Definition [24] A braided fusion category $\mathcal{C}$ is called almost non-degenerate if $Z_{2}(\mathcal{C}) \simeq S$ Vect. (Equivalently, $Z_{2}(\mathcal{C})$ has precisely one non-trivial simple object $X$ satisfying $X^{2} \cong \mathbf{1}$ and $\theta_{X}=-\mathrm{id}$.)

Almost modular categories will briefly be mentioned again at the end of this review, when the recent results of 24] will be touched upon.

The above considerations have an important generalization: Let $\mathcal{S} \subset \mathcal{C}$ be an arbitrary full symmetric fusion subcategory of the pre-modular category $\mathcal{C}$. For simplicity, we restrict to the case where $\mathcal{S}$ is even and thus equivalent (as a BTC) to the representation category of a finite group $G$. Let $A \in \mathcal{S}$ be the regular algebra. Since the latter contains all simple objects of $\mathcal{S}$ as direct summands, we have $A \in Z_{2}(\mathcal{C})$ if and only if $\mathcal{S} \subset Z_{2}(\mathcal{C})$. If these equivalent conditions are satisfied, ${ }_{A} \mathcal{C}$ is braided and $F_{A}: \mathcal{C} \rightarrow{ }_{A} \mathcal{C}$ is a braided functor that trivializes the subcategory $\mathcal{S} \subset \mathcal{C}$. In that case, one finds $Z_{2}\left({ }_{A} \mathcal{C}\right) \simeq{ }_{A}\left(Z_{2}(\mathcal{C})\right)$, which is trivial if and only if $\mathcal{S}=Z_{2}(\mathcal{C})$, recovering the previous result about modularization.

However, it is interesting to drop the requirement $\mathcal{S} \subset Z_{2}(\mathcal{C})$. Independently of this assumption, one finds that ${ }_{A} \mathcal{C}$ is a fusion category and $F_{A}$ a surjective tensor functor. Furthermore, the group $G$ acts on the module category ${ }_{A} \mathcal{C}$ (by monoidal self-equivalences) and one has $\left({ }_{A} \mathcal{C}\right)^{G} \simeq \mathcal{C}$. When $\mathcal{S} \not \subset Z_{2}(\mathcal{C})$, there exists no braiding on ${ }_{A} \mathcal{C}$ rendering $F_{A}$ braided, but we have seen that there is a braided functor $\widehat{F_{A}}: \mathcal{C} \rightarrow Z_{1}\left({ }_{A} \mathcal{C}\right)$. In this specific situation, one can prove more: There is a $G$-grading on ${ }_{A} \mathcal{C}$, i.e. a map $\partial$ from the class of simple objects to $G$, constant on isomorphism classes and satisfying $\partial(X \otimes Y)=\partial X \cdot \partial Y$. (As a consequence, $\partial \mathbf{1}=e$ and $\partial \bar{X}=(\partial X)^{-1}$.) If $\mathcal{D}$ is a $G$-graded category and $g \in G$, we denote by $\mathcal{D}_{g}$ the full subcategory whose objects are direct sums of simple objects $X$ with $\partial X=g$. Now we have

$$
\left({ }_{A} \mathcal{C}\right)_{e}={ }_{A}\left(C_{\mathcal{C}}(\mathcal{S})\right)={ }_{A} \mathcal{C}^{0} .
$$

The action of $G$ on ${ }_{A} \mathcal{C}$ and the $G$-grading are connected by the identity $\partial(g X)=g(\partial X) g^{-1}$, which is why ${ }_{A} \mathcal{C}$ is called a $G$-crossed category. While ${ }_{A} \mathcal{C}$ does not admit a braiding (in the usual sense), it does admit a generalized braiding that takes the grading and the $G$-action into account: For every $Y \in{ }_{A} \mathcal{C}$ and every $X \in\left({ }_{A} \mathcal{C}\right)_{g}$, there is an isomorphism $c_{X, Y}: X \otimes Y \rightarrow(g Y) \otimes X$ satisfying natural generalizations of the axioms for a braiding. Thus ${ }_{A} \mathcal{C}$ is a braided $G$-crossed category. Conversely, if $\mathcal{D}$ is a braided $G$-crossed fusion category then $\mathcal{C}=\mathcal{D}^{G}$ is an ordinary braided fusion category containing $\operatorname{Rep} G$ as a full subcategory, and if $A$ is the regular algebra in $\operatorname{Rep} G$ then ${ }_{A} \mathcal{C} \simeq \mathcal{D}$.

Most of these results are due to [53, 64, 68 in the case of spherical fusion categories. For a (somewhat) more extensive review than the one above, cf. 70. A much longer discussion, including generalizations to not-necessarily-spherical fusion categories and proofs pf precise 2-equivalences between categories of braided $G$-crossed fusion categories and braided fusion categories containing $\operatorname{Rep} G$, cf. [29].

Using the above results one can prove [70] the following result concerning Conjecture 5.23

\subsection{THEOREM The following are equivalent:}

(i) Conjecture 5.23 is true for every braided fusion category $\mathcal{C}$ whose symmetric center $Z_{2}(\mathcal{C}$ ) is even (and therefore equivalent to $\operatorname{Rep} G$ for a finite group $G$ ).

(ii) For every modular category $\mathcal{M}$ acted upon by a finite group $G$ there is a braided crossed $G$-category $\mathcal{E}$ with full $G$-spectrum and a $G$-equivariant equivalence $\mathcal{E}_{e} \simeq \mathcal{M}$.

We close this section by pointing out that the above results have applications to the orbifold construction in conformal field theory, cf. [70, Section 6] and references given there.

\section{The braided center of a fusion category}

As mentioned earlier, to every finite dimensional Hopf algebra $H$ one can associate 28] a finite dimensional quasi-triangular Hopf algebra $(D(H), R)$, Drinfeld's 'quantum double' of $H$. (The construction is not restricted to finite dimensional algebras, but it becomes more technical if $H$ is infinite dimensional and less relevant for the purposes of this review.) The case where $H$ is the group algebra of a finite group $G$ is denoted $D(G)$ and can be described very explicitly. In particular, the braided category $D(G)$-Mod is modular [1].

More generally, (i) semisimplicity of $D(H)$ is equivalent to (ii) semisimplicity of $H$ and of the dual Hopf algebra $\widehat{H}$ and to (iii) $S_{H}^{2}=$ id and $\operatorname{dim} H \neq 0$ in the ground field $k$. Under these assumptions, $D(H)$-Mod is modular [31. (Cf. [66, Appendix] for an alternative approach.)

Since the module category of a Hopf algebra $H$ satisfying the above conditions is a spherical fusion category satisfying $\operatorname{dim} D(H)$-Mod $=\operatorname{dim}_{k} H \neq 0$, the following results proven in [66] generalize those on $D(G)$ and $D(H)$ : 
7.1 Theorem Let $k$ be an algebraically closed field and $\mathcal{C}$ a $k$-linear semisimple spherical category satisfying $\operatorname{dim} \mathcal{C} \neq 0$. Then

(i) $Z_{1}(\mathcal{C})$ is semisimple.

(ii) $Z_{1}(\mathcal{C})$ has a natural spherical structure inherited from $\mathcal{C}$ and $\operatorname{dim} Z_{1}(\mathcal{C})=(\operatorname{dim} \mathcal{C})^{2}$.

(iii) $Z_{1}(\mathcal{C})$ is non-degenerate, thus modular.

(iv) The Gauss sums 5.1) of $Z_{1}(\mathcal{C})$ are given by $\Omega^{ \pm}\left(Z_{1}(\mathcal{C})\right)=\operatorname{dim} \mathcal{C}$.

(v) The forgetful functor $K: Z_{1}(\mathcal{C}) \rightarrow \mathcal{C}$ has a two-sided adjoint.

(vi) If $\mathcal{C}$ already is modular, then the braided tensor functor $H: \mathcal{C} \otimes \widetilde{\mathcal{C}} \rightarrow Z_{1}(\mathcal{C})$ is an equivalence.

(vii) If $\mathcal{C}_{1}, \mathcal{C}_{2}$ satisfy the above assumptions and $\mathcal{C}_{1} \approx \mathcal{C}_{2}$ (monoidal Morita equivalence of [65]) then $Z_{1}\left(\mathcal{C}_{1}\right) \simeq$ $Z_{1}\left(\mathcal{C}_{2}\right)$ (braided equivalence).

7.2 Remark 1. These results have been generalized to not necessarily spherical fusion categories, cf. 34. Cf. also [19] for a more conceptual approach in terms of Hopf monads.

2. (vii) is an easy consequence of the definitions and a result in 83 . The converse of (vii) is also true, cf. [33, Theorem 3.1].

3. In view of (vi) and the considerations in Section 4.3 , one could take the equivalence $Z_{1}(\mathcal{C}) \simeq \mathcal{C} \otimes \widetilde{\mathcal{C}}$ as alternative definition of modularity/non-degeneracy.

4. Statement (iii) means that $Z_{2}\left(Z_{1}(\mathcal{C})\right)$ is trivial for any spherical fusion category $\mathcal{C}$. This should be compared with the other results of the type 'the center of a center is trivial' mentioned in Remark 4.6. It is tempting to conjecture that this holds more generally in the context of centers in higher category theory.

5. Corollary (vi) implies that every modular category $\mathcal{C}$ arises as a direct factor of the braided center of some fusion category $\mathcal{D}$ : Just take $\mathcal{D}=\mathcal{C}$. This is interesting since the braiding of $\mathcal{C}$ is not used in $\operatorname{defining} Z_{1}(\mathcal{C})$. However, it seems pointless to reduce the classification of modular categories to the classification of fusion categories, since there are many more of the latter and there is no hope of classification. A more promising approach to 'classifying' modular categories will be discussed in the last section.

6. By (i)-(iii), the braided center construction gives rise to many modular categories. However, not every modular category $\mathcal{C}$ is equivalent to some $Z_{1}(\mathcal{D})$. This follows already from (iv) and the fact that there are modular categories whose two Gauss sums are not equal. A criterion for recognizing whether a modular category is of the form $Z_{1}(\mathcal{D})$ will be given below. Even when a modular category $\mathcal{C}$ does not satisfy this, one can often find fusion categories $\mathcal{D}$ smaller than $\mathcal{C}$ such that $Z_{1}(\mathcal{D})$ has $\mathcal{C}$ as a direct factor.

We now turn to the question of recognizing the modular categories that are of the form $Z_{1}(\mathcal{D})$ for $\mathcal{D}$ fusion, which has been solved quite recently, cf. [29, 23]. As mentioned in Section 4.4 a commutative (étale, connected) algebra $A$ in a braided fusion category $\mathcal{C}$ gives rise to a braided tensor functor $F_{A}: \mathcal{C} \rightarrow Z_{1}\left({ }_{A} \mathcal{C}\right)$. Under very weak conditions $F_{A}$ is faithful. In general, $F_{A}$ need not be full, but it is so when $\mathcal{C}$ is non-degenerate. This can be shown either by direct - and tedious - computation of $\operatorname{Hom}_{Z_{1}(\mathcal{C})}\left(F_{A}(X), F_{A}(Y)\right)$ or by invoking Proposition 5.24 . Thus if $\mathcal{C}$ is non-degenerate, $F_{A}: \mathcal{C} \rightarrow Z_{1}\left({ }_{A} \mathcal{C}\right)$ is an embedding of braided fusion categories, which by Theorem 5.19 (iv) gives rise to a direct factorization. The complementary factor $C_{Z_{1}(\mathcal{C})}\left(F_{A}(\mathcal{C})\right)^{\prime}$ can be identified using the result of Schauenburg mentioned in the last paragraph of Section 4.4. Recall that if $\mathcal{C}$ is non-degenerate braided fusion then we have the braided equivalence $\mathcal{C} \otimes \widetilde{\mathcal{C}} \stackrel{F_{1} \otimes F_{2}}{\longrightarrow} Z_{1}(\mathcal{C})$. If $A \in \mathcal{C}$ is a commutative algebra then $B=F_{2}(A) \in Z_{1}(\mathcal{C})$ is a commutative algebra and $\underline{B}=A$. The equivalence $Z_{1}(\mathcal{C}) \rightarrow \mathcal{C} \otimes \widetilde{\mathcal{C}}$ maps $B$ to $1 \otimes A$. Combining with Schauenburg's result, we have

$$
Z_{1}\left({ }_{A} \mathcal{C}\right)=Z_{1}(\underline{B} \mathcal{C}) \simeq{ }_{B} Z_{1}(\mathcal{C})^{0} \simeq{ }_{(1 \otimes A)}(\mathcal{C} \otimes \widetilde{\mathcal{C}})^{0}=\mathcal{C} \nabla_{A} \widetilde{\mathcal{C}}^{0}=\mathcal{C} \otimes \widetilde{{ }_{A} \mathcal{C}^{0}}
$$

Thus, if $\mathcal{C}$ is non-degenerate braided and $A \in \mathcal{C}$ a connected étale algebra, there is a braided equivalence

$$
Z_{1}\left({ }_{A} \mathcal{C}\right) \simeq \mathcal{C} \otimes \widetilde{{ }_{A} \mathcal{C}^{0}} .
$$

Since one can prove, cf. [23], that $\mathcal{C}=Z_{1}(\mathcal{D})$ contains a connected étale algebra such that ${ }_{A} \mathcal{C}^{0}$ is trivial, one arrives at the following characterization of Drinfeld centers of fusion categories:

7.3 Theorem $A$ non-degenerate braided fusion category $\mathcal{C}$ is equivalent to $Z_{1}(\mathcal{D})$, where $\mathcal{D}$ is a fusion category, if and only if there is a connected étale algebra $A$ in $\mathcal{C}$ such that ${ }_{A} \mathcal{C}^{0}$ is trivial. In this case, one can take $\mathcal{D}={ }_{A} \mathcal{C}$. 
In Section 6, we defined fusion categories graded by a finite group. One can ask how a $G$-grading on a fusion category $\mathcal{C}$ is reflected in the center $Z_{1}(\mathcal{C})$. This was clarified in [43, where the following is proven:

7.4 Theorem [43, Theorem 3.5] Let $\mathcal{C}$ be $G$-graded with degree zero component $\mathcal{C}_{e}$. Then the relative Drinfeld center $Z_{1}\left(\mathcal{C}, \mathcal{C}_{e}\right)$ mentioned in Remark 4.6 .2 (which is monoidal but not braided) has a natural structure of braided $G$-crossed category, and there is an equivalence

$$
Z_{1}\left(\mathcal{C}, \mathcal{C}_{e}\right)^{G} \simeq Z_{1}(\mathcal{C})
$$

of braided categories.

The interest of this theorem derives from the fact that the relative center $Z_{1}\left(\mathcal{C}, \mathcal{C}_{e}\right)^{G}$ may be easier to determine than the full $Z_{1}(\mathcal{C})$.

We close this section with an important application of the braided center $Z_{1}$ and of Theorem 7.1 to topology. Since $Z_{1}(\mathcal{C})$ is modular when $\mathcal{C}$ is fusion, it gives rise to a Reshetikhin-Turaev TQFT [81, 86, 87. It is natural to ask whether there is a more direct construction of this TQFT in terms of the spherical category $\mathcal{C}$. In fact, shortly after the Reshetikhin-Turaev construction, Turaev and Viro [90, 87, proposed a construction of $2+1$ dimensional TQFTs in terms of triangulations and 'state-sums' rather than surgery. While being fundamentally different from the RT-approach, the TV construction still required a modular category as input. It was realized by various authors that the construction of a state-sum TQFT actually does not require a braiding and that a spherical fusion category suffices as input datum, cf. in particular [9]. (The same observation was also made by Ocneanu and by S. Gelfand and Kazhdan.) This made it natural to conjecture that the state-sum TQFT of [9] associated with a spherical fusion category $\mathcal{C}$ is isomorphic to the surgery TQFT associated with the modular category $Z_{1}(\mathcal{C})$. This conjecture was proven in 2010, independently by Turaev and Virelizier [89, based on extensive previous work by Bruguières and Virelizier [19, 20, and by Balsam and Kirillov [7, 6].

\section{The Witt group of modular categories}

The results in this subsection are from [23]. They are motivated by the desire to 'classify' modular categories (or non-degenerate braided fusion categories). This is a rather hopeless project since, by Theorem $7.1, Z_{1}(\mathcal{D})$ is modular whenever $\mathcal{D}$ is a spherical fusion category and since there is little hope of classifying fusion categories. (Recall that, e.g., every semisimple Hopf algebra gives rise to a fusion category.) The fact that Morita equivalent fusion categories have equivalent Drinfeld centers reduces the problem only marginally.

This leads to the idea of considering categories of the form $Z_{1}(\mathcal{D})$ with $\mathcal{D}$ fusion as 'trivial' and of classifying modular categories (or non-degenerate braided fusion categories) 'up to centers'. The following definition provides a rigorous way of doing this.

8.1 Definition Two non-degenerate braided fusion categories $\mathcal{C}_{1}, \mathcal{C}_{2}$ are called Witt equivalent if there are fusion categories $\mathcal{D}_{1}, \mathcal{D}_{2}$ such that there is a braided equivalence $\mathcal{C}_{1} \otimes Z_{1}\left(\mathcal{D}_{1}\right) \simeq \mathcal{C}_{2} \otimes Z_{1}\left(\mathcal{D}_{2}\right)$.

Witt equivalence obviously is coarser than braided equivalence, and it is not hard to show that it is an equivalence relation. In fact, the Witt classes form a set $W_{M}$ that actually is countable. Denoting the Witt equivalence class of $\mathcal{C}$ by $[\mathcal{C}], W_{M}$ becomes an abelian monoid via $\left[\mathcal{C}_{1}\right] \cdot\left[\mathcal{C}_{2}\right]:=\left[\mathcal{C}_{1} \otimes \mathcal{C}_{2}\right]$ with unit $\mathbf{1}_{W_{M}}=[$ Vect]. Up to this point, analogous results hold for the set of braided equivalence classes of non-degenerate braided fusion categories, of which $W_{M}$ is the quotient monoid under the identification $\left[Z_{1}(\mathcal{D})\right]=[$ Vect $]$ for each fusion category $\mathcal{D}$. But $W_{M}$ has one crucial additional property: It is a group. Namely, defining $[\mathcal{C}]^{-1}:=[\widetilde{\mathcal{C}}]$, we have

$$
[\mathcal{C}] \cdot[\mathcal{C}]^{-1}=[\mathcal{C}] \cdot[\widetilde{\mathcal{C}}]=[\mathcal{C} \otimes \widetilde{\mathcal{C}}]=\left[Z_{1}(\mathcal{C})\right]=\mathbf{1}_{W_{M}},
$$

where the penultimate identity crucially depends on (vii) of Theorem 7.1. Therefore, $W_{M}$ is called the Witt group. (Actually, there are three Witt groups, defined in terms of non-degenerate braided fusion categories, modular categories and unitary modular categories, respectively.)

By 7.1 , an étale algebra $A \in \mathcal{C}$ gives rise to a braided equivalence $Z_{1}\left({ }_{A} \mathcal{C}\right) \simeq \mathcal{C} \otimes \widetilde{{ }_{A} \mathcal{C}^{0}}$ and therefore to the identity $[\mathcal{C}]=\left[{ }_{A} \mathcal{C}^{0}\right]$ in the Witt group. Using the fact that $Z_{1}(\mathcal{D})$, where $\mathcal{D}$ is fusion, contains an étale algebra $A$ such that ${ }_{A} \mathcal{C}^{0}$ is trivial, the following is not hard to show, cf. [23]:

8.2 TheOREm Let $\mathcal{C}_{1}, \mathcal{C}_{2}$ be non-degenerate braided fusion categories. Then the following are equivalent:

(i) $\left[\mathcal{C}_{1}\right]=\left[\mathcal{C}_{2}\right]$, i.e. $\mathcal{C}_{1}$ and $\mathcal{C}_{2}$ are Witt equivalent. 
(ii) There is a fusion category $\mathcal{D}$ such that $\mathcal{C}_{1} \otimes \widetilde{\mathcal{C}_{2}} \simeq Z_{1}(\mathcal{D})$.

(iii) There is a connected étale algebra $A \in \mathcal{C}_{1} \otimes \widetilde{\mathcal{C}_{2}}$ such that ${ }_{A}\left(\mathcal{C}_{1} \otimes \widetilde{\mathcal{C}_{2}}\right)^{0}$ is trivial.

(iv) There exist a non-degenerate braided fusion category $\mathcal{C}$, connected étale algebras $A_{1}, A_{2} \in \mathcal{C}$ and braided equivalences $\mathcal{C}_{1} \simeq{ }_{A_{1}} \mathcal{C}^{0}, \mathcal{C}_{2} \simeq{ }_{A_{2}} \mathcal{C}^{0}$.

(v) There exist connected étale algebras $A_{1} \in \mathcal{C}_{1}, A_{2} \in \mathcal{C}_{2}$ and a braided equivalence ${ }_{A_{1}} \mathcal{C}_{1}^{0} \simeq{ }_{A_{2}} \mathcal{C}_{2}^{0}$.

This shows that Witt equivalence could have been defined in terms of dyslectic module categories instead of invoking the braided center $Z_{1}$. This latter approach has a 'physical' interpretation: Consider a rational chiral conformal field theory $\mathcal{A}$, either as a $\left(C_{2}\right.$-cofinite) vertex operator algebra or in terms of von Neumann algebras indexed by intervals on $S^{1}$, as e.g. in [94, 96, 151. As mentioned earlier, in both settings there is a proof of modularity of the representation category $\operatorname{Rep} \mathcal{A}$. Furthermore, in both settings, there is a notion of 'finite extension' (or conformal extension) and one can prove that the finite extensions $\mathcal{B} \supset \mathcal{A}$ are classified by the connected étale algebras $A \in \operatorname{Rep} \mathcal{A}$ in such way that $\operatorname{Rep} \mathcal{B}={ }_{A}(\operatorname{Rep} \mathcal{A})^{0}$ when the extension $\mathcal{B} \supset \mathcal{A}$ corresponds to the algebra $A \in \operatorname{Rep} \mathcal{A}$. (Cf. [54 and 71 for proof sketches.) This fact implies that we have $[\operatorname{Rep} \mathcal{B}]=[\operatorname{Rep} \mathcal{A}]$ for any finite extension $\mathcal{B} \supset \mathcal{A}$ of rational chiral CFTs.

A (not very precise) folk conjecture in conformal field theory, cf. e.g. 63], states that every modular category (to the extent that it is realized by a CFT) can be obtained from the modular categories arising from WZW models combined and a certain set of 'constructions' (like orbifold and coset constructions). Now, the WZW categories coincide with the representation categories of quantum groups at root-of-unity deformation parameter. Thus if one accepts that the above constructions amount to passing to finite index subtheories and extensions, one arrives at the following mathematical formulation of the Moore-Seiberg conjecture:

8.3 Conjecture The Witt group $W_{M}$ is generated by the classes $[\mathcal{C}(\mathfrak{g}, q)]$ of the quantum group categories $\mathcal{C}(\mathfrak{g}, q)$, where $\mathfrak{g}$ is a simple Lie algebra and $q$ a root of unity.

The only evidence for the conjecture so far is that there are no counterexamples! While there are fusion categories that are 'exotic' in the sense of having no (known) connection with finite group theory or Lie theory, no modular categories are known that are 'genuinely exotic' in the sense of not being (related to) Drinfeld centers of exotic fusion categories. However, the existing classification of conformal extensions provides a large and presumably complete set of relations in the subgroup of the Witt group generated by the classes $[\mathcal{C}(\mathfrak{g}, q)]$. While the full group $W_{M}$ is not understood, a close relative, to wit the Witt group of almost non-degenerate braided fusion categories, has been computed recently, cf. [24.

The circle of ideas around Witt equivalence is also relevant for the construction of two-dimensional CFTs from a pair of chiral ('one-dimensional') CFTs. The relevant mathematical structure seems to be the following:

8.4 Definition Let $\mathcal{C}_{1}, \mathcal{C}_{2}$ be modular categories. A modular invariant for $\left(\mathcal{C}_{1}, \mathcal{C}_{2}\right)$ is a triple $\left(A_{1}, A_{2}, E\right)$, where $A_{1} \in \mathcal{C}_{1}, A_{2} \in \mathcal{C}_{2}$ are connected étale algebras and $E:{ }_{A_{1}} \mathcal{C}_{1}^{0} \rightarrow{ }_{A_{2}} \mathcal{C}_{2}^{0}$ is a braided equivalence.

In view of Theorem 8.2 , it is clear that a modular invariant for $\left(\mathcal{C}_{1}, \mathcal{C}_{2}\right)$ exists if and only if $\mathcal{C}_{1}$ and $\mathcal{C}_{2}$ are Witt equivalent. But more can be said (stated in [71] and proven in [24]):

8.5 Proposition If $\mathcal{C}_{1}, \mathcal{C}_{2}$ are modular, there is a bijection (modulo natural equivalence relations) between modular invariants $\left(A_{1}, A_{2}, E\right)$ for $\left(\mathcal{C}_{1}, \mathcal{C}_{2}\right)$ and connected étale algebras $A \in \mathcal{C}_{1} \otimes \widetilde{\mathcal{C}_{2}}$ such that ${ }_{A}\left(\mathcal{C}_{1} \otimes \widetilde{\mathcal{C}_{2}}\right)^{0}$ is trivial $\left(\Leftrightarrow d(A)=\sqrt{\operatorname{dim} \mathcal{C}_{1} \cdot \operatorname{dim} \mathcal{C}_{2}}\right)$.

A related result proven in [40] involves non-commutative algebras in a modular category. The fact that an algebra over a field has a center, which is a commutative algebra, generalizes to braided spherical categories. But since the definition of the center of an algebra $A$ in a braided category $\mathcal{C}$ involves the braiding, there will actually be two centers $Z_{L}(A), Z_{R}(A)$, depending on the use of $c$ or $\widetilde{c}$. One finds

8.6 TheOrem [40] Let $A$ be a separable connected algebra in a modular category $\mathcal{C}$. Then the centers $Z_{L}(A), Z_{R}(A)$ are connected étale and there is an equivalence

$$
E:{ }_{Z_{L}(A)} \mathcal{C}^{0} \rightarrow{ }_{Z_{R}(A)} \mathcal{C}^{0}
$$

of braided categories. (Thus $\left(Z_{L}(A), Z_{R}(A), E\right)$ is a modular invariant in the sense of Definition 8.4 .)

Every modular invariant arises in this way from a separable connected algebra $A$ in $\mathcal{C}$, cf. [56]. 
8.7 REMARK 1. The categorical constructions in [40] were inspired by analogous constructions in an operator algebraic context, cf. [17, and conjectures in [77.

2. In the series of papers [41, a construction of "topological two-dimensional CFTs" was given taking a modular category and a separable connected algebra in it as a starting point. (The quotation marks refer to the fact that a CFT is more than a TQFT: It involves infinite dimensional Hilbert spaces, trace class operators, analytic characters, etc.)

\section{References}

[1] D. Altschuler, A. Coste: Invariants of 3-manifolds from finite groups. In: Proc. XXth Int. Conf. Diff. Geom. Meth. in Theor. Phys., New York, 1991, pp. 219-233, World Scientific, 1992.

[2] M. Atiyah: Topological quantum field theories. Publ. Math. IHES 68, 175-186 (1989).

[3] J. C. Baez, J. Dolan: Categorification. In: E. Getzler, M. Kapranov (eds.): Higher Category Theory. Contemp. Math. 230, 1-36 (1998).

[4] J. C. Baez; J. P. May (eds.): Towards higher categories. Springer Verlag, 2010.

[5] B. Bakalov, A. Kirillov Jr.: Lectures on tensor categories and modular functors. American Mathematical Society, 2001.

[6] B. Balsam: Turaev-Viro invariants as an extended TQFT II \& III. arXiv:1010.1222 \& 1012.0560.

[7] B. Balsam, A. Kirillov Jr.: Turaev-Viro invariants as an extended TQFT. arXiv:1004.1533.

[8] P. Bantay: The kernel of the modular representation and the Galois action in RCFT. Comm. Math. Phys. 233, 423-438 (2003).

[9] J. W. Barrett, B. W. Westbury: Invariants of piecewise-linear 3-manifolds. Trans. Amer. Math. Soc. 348, 3997-4022 (1996).

[10] J. W. Barrett, B. W. Westbury: Spherical categories. Adv. Math. 143, 357-375 (1999).

[11] H. Baumgärtel, F. Lledó: Duality of compact groups and Hilbert $\mathrm{C}^{*}$-systems for $\mathrm{C}^{*}$-algebras with a nontrivial center. Int. J. Math. 15, 759-812 (2004).

[12] A. Beliakova, C. Blanchet: Skein construction of idempotents in Birman-Murakami-Wenzl algebras. Math. Ann. 321, 347-373 (2001).

[13] A. Beliakova, C. Blanchet: Modular categories of types B, C and D. Comment. Math. Helv. 76, 467-500 (2001).

[14] J. Benabou: Catégories avec multiplication. C. R. Acad. Sci. Paris 256, 1887-1890 (1963).

[15] R. Bezrukavnikov: On tensor categories attached to cells in affine Weyl groups. In: Representation theory of algebraic groups and quantum groups, p. 69-90, Adv. Stud. Pure Math. 40, Math. Soc. Japan, 2004.

[16] C. Blanchet: Hecke algebras, modular categories and 3-manifolds quantum invariants. Topology 39, 193$223(2000)$.

[17] J. Böckenhauer, D. E. Evans, Y. Kawahigashi: On $\alpha$-induction, chiral generators and modular invariants for subfactors. Comm. Math. Phys. 208, 429-487 (1999). Chiral structure of modular invariants for subfactors. Comm. Math. Phys. 210, 733-784 (2000).

[18] A. Bruguières: Catégories prémodulaires, modularisations et invariants de variétés de dimension 3. Math. Ann. 316, 215-236 (2000).

[19] A. Bruguières, A. Virelizier: Hopf monads. Adv. Math. 215, 679-733 (2007). Categorical centers and Reshetikhin-Turaev invariants. Acta Math. Vietnam. 33, 255-277 (2008). Quantum double of Hopf monads and categorical centers. Trans. AMS. 364, 1225-1279 (2012).

[20] A. Bruguières, S. Lack, A. Virelizier: Hopf monads on monoidal categories. Adv. Math. 227, 745-800 (2011). 
[21] V. Chari, A. Pressley: A guide to quantum groups. Cambridge University Press, 1995.

[22] A. A. Davydov: Monoidal categories. J. Math. Sci. (New York) 88, 457-519 (1998).

[23] A. Davydov, M. Müger, D. Nikshych, V. Ostrik: The Witt group of non-degenerate braided fusion categories. To appear in J. Reine Angew. Math. (arXiv:1009.2117.)

[24] A. Davydov, D. Nikshych, V. Ostrik: On the structure of the Witt group of braided fusion categories. arXiv:1109.5558.

[25] P. Deligne: Catégories tannakiennes. In: P. Cartier et al. (eds.): Grothendieck Festschrift, vol. II, pp. 111-195. Birkhäuser Verlag 1991.

[26] S. Doplicher, J. E. Roberts: A new duality theory for compact groups. Invent. Math. 98, 157-218 (1989).

[27] C. Douglas, C. Schommer-Pries, N. Snyder: work in progress. Cf. the slides at http://ncatlab.org/nlab/files/DSSFusionSlides.pdf

[28] V. G. Drinfeld: Quantum groups. In: Proc. Int. Congr. Math, Berkeley 1986.

[29] V. Drinfeld, S. Gelaki, D. Nikshych, V. Ostrik: Group-theoretical properties of nilpotent modular categories. arXiv:0704.0195.

[30] V. Drinfeld, S. Gelaki, D. Nikshych, V. Ostrik: On braided fusion categories. I. Selecta Math. (N.S.) 16, 1-119 (2010).

[31] P. Etingof, S. Gelaki: Some properties of finite-dimensional semisimple Hopf algebras. Math. Res. Lett. 5, 191-197 (1998).

[32] P. Etingof, D. Nikshych, V. Ostrik: On fusion categories. Ann. Math. 162, 581-642 (2005).

[33] P. Etingof, D. Nikshych, V. Ostrik: Weakly group-theoretical and solvable fusion categories. Adv. Math. 226, 176-205 (2011).

[34] P. Etingof, V. Ostrik: Finite tensor categories. Mosc. Math. J. 4, 627-654, 782-783 (2004).

[35] K. Fredenhagen, K.-H. Rehren, B. Schroer: Superselection sectors with braid group statistics and exchange algebras I. General theory. Comm. Math. Phys. 125, 201-226 (1989). II. Geometric aspects and conformal covariance. Rev. Math. Phys. 1992, Special Issue, 113157.

[36] M. H. Freedman, A. Kitaev, M. J. Larsen, Z. Wang: Topological quantum computation. Mathematical challenges of the 21st century. Bull. Amer. Math. Soc. 40, 31-38 (2003).

[37] P. Freyd, D. Yetter: Braided compact closed categories with applications to low-dimensional topology. Adv. Math. 77, 156-182 (1989).

[38] P. Freyd, D. Yetter: Coherence theorems via knot theory. J. Pure Appl. Alg. 78, 49-76 (1992).

[39] P. Freyd, D. Yetter, J. Hoste, W. B. R. Lickorish, K. Millett, A. Ocneanu: A new polynomial invariant of knots and links. Bull. Amer. Math. Soc. 12, 239-246 (1985).

[40] J. Fröhlich, J. Fuchs, I. Runkel, C. Schweigert: Correspondences of ribbon categories. Adv. Math. 199, 192-329 (2006).

[41] J. Fuchs, I. Runkel, C. Schweigert: TFT construction of RCFT correlators I-V. Nuclear Phys. B646, 353497 (2002), B678, 511-637 (2004), B694, 277-353 (2004), B715, 539-638 (2005); Theory Appl. Categ. 16, 342-433 (2006).

[42] S. Gelaki, D. Nikshych: Nilpotent fusion categories. Adv. Math. 217, 1053-1071 (2008).

[43] S. Gelaki, D. Naidu, D. Nikshych: Centers of graded fusion categories. Alg. Num. Th. 3, 959-990 (2009).

[44] R. Haag: Local quantum physics. Springer, 192, 2996.

[45] Y.-Z. Huang: Vertex operator algebras, the Verlinde conjecture, and modular tensor categories. Proc. Natl. Acad. Sci. USA 102, 5352-5356 (2005). 
[46] V. F. R. Jones: A polynomial invariant for knots via von Neumann algebras. Bull. Amer. Math. Soc. 12, 103-111 (1985).

[47] A. Joyal, R. Street: Tortile Yang-Baxter operators in tensor categories. J. Pure Appl. Alg. 71, 43-51 (1991).

[48] A. Joyal, R. Street: Braided tensor categories. Adv. Math. 102, 20-78 (1993). (Preprint dates back to 1986.)

[49] C. Kassel: Quantum groups. Springer GTM 155, 1995.

[50] C. Kassel, V. G. Turaev: Chord diagram invariants of tangles and graphs. Duke Math. J. 92, 497-552 (1998).

[51] Y. Kawahigashi, R. Longo, M. Müger: Multi-interval subfactors and modularity of representations in conformal field theory. Comm. Math. Phys. 219, 631-669 (2001).

[52] D. Kazhdan, G. Lusztig: Tensor structures arising from affine Lie algebras I-IV. J. Amer. Math. Soc. 6, 905-947, 949-1011 (1993); 7, 335-381, 383-453. (1994).

[53] A. Kirillov Jr.: Modular categories and orbifold models I \& II. Comm. Math. Phys. 229, 309-335 (2002) \& math.QA/0110221. On $G$-equivariant modular categories. math.QA/0401119.

[54] A. Kirillov Jr., V. Ostrik: On q-analog of McKay correspondence and ADE classification of $s l(2)$ conformal field theories. Adv. Math. 171, 183-227 (2002).

[55] A. Kitaev: Fault-tolerant quantum computation by anyons. Ann. Physics 303, 230 (2003).

[56] L. Kong, I. Runkel: Cardy algebras and sewing constraints. I. Comm. Math. Phys. 292, 871-912 (2009).

[57] R. Longo, J. E. Roberts: A theory of dimension. K-Theory 11, 103-159 (1997).

[58] S. Mac Lane: Natural associativity and commutativity. Rice Univ. Studies 49, 28-46 (1963).

[59] S. Mac Lane: Categories for the working mathematician. 2nd ed. Springer Verlag, 1998.

[60] S. Majid: Representations, duals and quantum doubles of monoidal categories. Rend. Circ. Mat. Palermo Suppl. 26, 197-206 (1991).

[61] S. Majid: Foundations of quantum group theory. Cambridge University Press, 1995.

[62] G. Moore, N. Seiberg: Classical and quantum conformal field theory. Comm. Math. Phys. 123, 177-254 (1989).

[63] G. Moore, N. Seiberg: Taming the conformal zoo. Phys. Lett. B220, 422-430 (1989).

[64] M. Müger: Galois theory for braided tensor categories and the modular closure. Adv. Math. 150, 151-201 (2000).

[65] M. Müger: From subfactors to categories and topology I. Frobenius algebras in and Morita equivalence of tensor categories. J. Pure Appl. Alg. 180, 81-157 (2003).

[66] M. Müger: From subfactors to categories and topology II. The quantum double of tensor categories and subfactors. J. Pure Appl. Alg. 180, 159-219 (2003).

[67] M. Müger: On the structure of modular categories. Proc. Lond. Math. Soc. 87, 291-308 (2003).

[68] M. Müger: Galois extensions of braided tensor categories and braided crossed G-categories. J. Alg. 277, 256-281 (2004).

[69] M. Müger: On the center of a compact group. Intern. Math. Res. Not. 2004:51, 2751-2756 (2004).

[70] M. Müger: On the structure of braided crossed $G$-categories. Appendix 5 in 88 .

[71] M. Müger: On Superselection theory of quantum fields in low dimensions. p. 496-503 in: P. Exner (ed.): Proceedings of the XVIth International Congress on Mathematical Physics. (Prague, August 3-8, 2009.) World Scientific, 2010. (arXiv:0909.2537) 
[72] M. Müger: Tensor categories: A selective guided tour. Rev. Un. Mat. Argent. 51, 95-163 (2010).

[73] P. Naaijkens: Localized endomorphisms in Kitaev's toric code on the plane. Rev. Math. Phys. 23, 347-373 (2011).

[74] D. Naidu, D. Nikshych, S. Witherspoon: Fusion subcategories of representation categories of twisted quantum doubles of finite groups. Int. Math. Res. Not. 2009, 41834219.

[75] C. Nayak, S. H. Simon, A. Stern, M. Freedman, S. Das Sarma: Non-abelian anyons and topological quantum computation. Rev. Mod. Phys. 80, 1083-1159 (2008).

[76] S.-H. Ng, P. Schauenburg: Congruence subgroups and generalized Frobenius-Schur indicators. Comm. Math. Phys. 300, 1-46 (2010).

[77] V. Ostrik: Module categories, weak Hopf algebras and modular invariants. Transf. Groups 8, 177-206 (2003).

[78] B. Pareigis: On braiding and dyslexia. J. Algebra 171, 413-425 (1995).

[79] A. Pressley, G. Segal: Loop groups. Oxford University Press, 1986.

[80] K.-H. Rehren: Braid group statistics and their superselection rules. In: D. Kastler (ed.): The algebraic theory of superselection sectors. Introduction and recent results. World Scientific, 1990.

[81] N. Yu. Reshetikhin, V. G. Turaev: Invariants of 3-manifolds via link polynomials and quantum groups. Invent. Math. 103, 547-598 (1991).

[82] E. Rowell: On a family of non-unitarizable ribbon categories. Math. Z. 250, 745-774 (2005).

[83] P. Schauenburg: The monoidal center construction and bimodules. J. Pure Appl. Alg. 158, 325-346 (2001).

[84] Y. Sommerhäuser, Y. Zhu: Hopf algebras and congruence subgroups. arXiv:0710.0705

[85] V. G. Turaev: Operator invariants of tangles, and R-matrices. Izv. Akad. Nauk SSSR 53, 1073-1107 (1989). (English translation: Math. USSR-Izv. 35, 411-444 (1990).)

[86] V. G. Turaev: Modular categories and 3-manifold invariants. Int. J. Mod. Phys. B6, 1807-1824 (1992).

[87] V. G. Turaev: Quantum invariants of knots and 3-manifolds. Walter de Gruyter, 1994.

[88] V. G. Turaev: Homotopy quantum field theory. (With appendices by M. Müger and A. Virelizier.) European Mathematical Society, 2010.

[89] V. G. Turaev, A. Virelizier: On two approaches to 3-dimensional TQFTs. arXiv:1006.3501.

[90] V. G. Turaev, O. Viro: State sum invariants of 3-manifolds and quantum $6 j$-symbols. Topology 31, 865-902 (1992).

[91] V. G. Turaev, H. Wenzl: Semisimple and modular categories from link invariants. Math. Ann. 309, 411-461 (1997).

[92] M. Wakimoto: Infinite dimensional Lie algebras. Amer. Math. Soc., 2001.

[93] Z. Wang: Topological quantum computation. Amer. Math. Soc., 2010.

[94] A. Wassermann: Operator algebras and conformal field theory III. Fusion of positive energy representations of $S U(N)$ using bounded operators. Invent. Math. 133, 467-538 (1998).

[95] E. Witten: Topological quantum field theory. Comm. Math. Phys. 117, 353-386 (1988), Quantum field theory and the Jones polynomial. Comm. Math. Phys. 121, 351-399 (1989).

[96] F. Xu: New braided endomorphisms from conformal inclusions. Comm. Math. Phys. 192, 349-403 (1998).

[97] S. Yamagami: Frobenius duality in $C^{*}$-tensor categories. J. Oper. Th. 52, 3-20 (2004).

[98] D. N. Yetter: Framed tangles and a theorem of Deligne on braided deformations of tannakian categories. Contemp. Math. 134, 325-350 (1992). 
[99] D. N. Yetter: Braided deformations of monoidal categories and Vassiliev invariants. Higher category theory (Evanston, IL, 1997), 117-134, Contemp. Math., 230, Amer. Math. Soc., Providence, RI, 1998.

[100] D. N. Yetter: Functorial knot theory. World Scientific, 2001. 